\title{
Passive scalars in turbulent channel flow at high Reynolds number
}

\author{
SERGIO PIROZZOLI, MATTEO BERNARDINI \\ and PAOLO ORLANDI
}

Dipartimento di Ingegneria Meccanica e Aerospaziale, Università di Roma 'La Sapienza' Via Eudossiana 18, 00184 Roma, Italy

(Received 17 November 2015)

We study passive scalars in turbulent plane channels at computationally high Reynolds number, which allows to observe previously unnoticed effects. The mean scalar profiles are found to obey a generalized logarithmic law which includes a linear correction term in the whole lower half-channel, and they follow a universal parabolic defect profile in the core region. This is consistent with recent findings regarding the mean velocity profiles in channel flow. The scalar variances also exhibit a near universal parabolic distribution in the core flow and hints of a sizeable log layer, unlike the velocity variances. The energy spectra highlight the formation of large scalar-bearing eddies with size proportional to the channel height which are caused by local production excess over dissipation, and which are clearly visible in the flow visualizations. Close correspondence of the momentum and scalar eddies is observed, the main difference being that the latter tend to form sharper gradients, which translates into higher scalar dissipation. Another notable Reynolds number effect is the decreased correlation of the passive scalar field with the vertical velocity field, which is traced to the reduced effectiveness of ejection events.

\section{Introduction}

Turbulent Poiseuille flow in a pressure-driven channel between parallel flat plates is the most widely studied case among canonical wall-bounded flows, being an ideal candidate for direct numerical simulation (DNS). Since the pioneering study of Kim et al. (1987) at low Reynolds number $\left(R e_{\tau} \approx 180\right.$, where $R e_{\tau}=h / \delta_{v}$, with $h$ the channel half-height and $\delta_{v}=\nu / u_{\tau}$ the viscous length scale), several studies have appeared in which Reynolds numbers are sufficient to observe a nascent logarithmic layer in the mean velocity profile (Moser et al. 1999; del Álamo \& Jiménez 2003; Bernardini et al. 2014; Lee \& Moser 2015). These studies have also allowed to observe the onset of structural changes in the overall flow organization, mainly related to the strengthening of outer-layer global modes which are found to superpose onto and modulate the energetic near-wall eddies (Hutchins \& Marusic 2007).

At least as important as the statistics of the velocity field are the statistics of passive scalars suspended in the fluid phase, which are representative for the behavior of diluted contaminants, and/or the temperature field under the assumption of low Mach numbers and small temperature differences (Monin \& Yaglom 1971; Cebeci \& Bradshaw 1984). It is well known that measurements of concentration of passive tracers and of small temperature differences are extremely complicated, and in fact available measurements of even basic passive scalar statistics are rather limited (Gowen \& Smith 1967; Kader 1981; Subramanian \& Antonia 1981; Nagano \& Tagawa 1988), mostly including the mean 
flow properties. Hence, DNS constitutes an invaluable tool for the study of passive scalar turbulence in Poiseuille flow. Early numerical experiments of passive scalars in turbulent channel flow (Kim \& Moin 1989) were carried out at $R e_{\tau}=180$, for $\operatorname{Pr}=0.1,0.71,2$ (here the molecular Prandtl number is defined as the ratio of the kinematic viscosity to the thermal diffusivity, $\operatorname{Pr}=\nu / \alpha$ ), by assuming uniform volumetric heating and constant wall properties. That study first showed close similarity in the near-wall region between the streamwise velocity and the scalar fields. Specifically, the scalar field was found to be organized into streaks whose size scales in wall units, with a correlation coefficient between streamwise velocity fluctuations and scalar fluctuations close to one. Numerical studies of scalar fields in a channel driven by differences at the two walls were carried out by Lyons et al. (1991); Wikström \& Johansson (1998); Kawamura et al. (2000). While the behavior in the near-wall region is similar between the forced case and the assigned scalar difference case, much stronger fluctuations are observed in the latter, owing to the formation of strong core eddies with an organization similar to the velocity field in turbulent Couette flow (Pirozzoli et al. 2014). Furthermore, low-Re simulations seemed to suggest influence of the wall state on the Karman constant for the scalar fields (Kawamura et al. 2000).

Studies of the effect of $\mathrm{Pr}$ in a wide range have been rather limited so far, mainly because of the need of extremely fine meshes to resolve the scalar fields, since the ratio of the Kolmogorov scale to the Batchelor scalar dissipative scale is supposed to be of the order of $\mathrm{Pr}^{-1 / 2}$ (Batchelor 1959; Tennekes \& Lumley 1972). Kawamura et al. (1998) performed DNS of channel flow at $R e_{\tau}=180$ with a constant time-averaged heat-flux condition, in the range of Prandtl number $0.025 \leqslant P r \leqslant 5$. The results proved validity of Kader's correlation for the passive scalar distribution (Kader 1981), and of heat flux correlations originally developed by Sleicher \& Rouse (1975) for pipe flow. Except for very low $P r$, the study also showed near universality of the distributions of the turbulent Prandtl number, with values of order unity throughout. Schwertfirm \& Manhart (2007) extended the study to $\operatorname{Pr} \approx 50$, basically observing a continuation of the lower- $\operatorname{Pr}$ results of Kawamura et al. (1998).

Reynolds number effects on passive scalar fields are difficult to study in DNS, owing to the rapid increase of the necessary computational resources. Kawamura et al. (1999) performed DNS of channel flow with forced scalar fields at $R e_{\tau}=180,395$, and $\operatorname{Pr}=0.025,0.2,0.71$. The main conclusion was that the log-law constant for the scalar field is roughly independent on the Reynolds number, with values of the Karman constant in the range $0.40 \leqslant k_{\theta} \leqslant 0.42$. Similarly, the turbulent Prandtl number was found to be weakly affected by Reynolds number variations. Abe et al. (2004b) further extended the range of Reynolds numbers to $R e_{\tau}=1020$, focusing the attention on the variation of the surface heat flux fluctuations. Those authors estimated a value of the Karman constant of $k_{\theta} \approx 0.43$, and observed an increase of the r.m.s. wall heat flux with $R e_{\tau}$, to a greater extent in the case of low $\mathrm{Pr}$. Based on the same DNS database, Abe \& Antonia (2009) quantified the degree of similarity between velocity and scalar fields and their corresponding derivatives near the wall through correlation coefficients, joint pdfs, Taylor series expansions, and instantaneous fields. That study indicated that impaired similarity between $u$ and $\theta$ occurs in regions where the magnitude of the streamwise pressure gradient is large due to the presence of near-wall vortical motions. The magnitudes of the scalar-velocity correlation coefficients were found to be relatively unaffected by the Reynolds number, although the authors explicitly stated that a firm conclusion could not be made due to the small range of $R e_{\tau}$ available at that time. Antonia et al. (2009) quantitatively analyzed similarities and differences between the fluctuating velocity vector and temperature fluctuations in turbulent channel flow. Strong similarities 


\begin{tabular}{lcccccccccc}
\hline Flow case & Line style & $R e_{b}$ & $R e_{\tau}$ & $N_{x}$ & $N_{y}$ & $N_{z}$ & $\Delta x^{+}$ & $\Delta y_{w}^{+}$ & $\Delta z^{+}$ & $T u_{\tau} / h$ \\
\hline P550 & dashed & 20063 & 548 & 1280 & 384 & 640 & 8.1 & 0.06 & 5.4 & 29.8 \\
P1000 & dash-dot & 39600 & 995 & 2560 & 512 & 1280 & 7.3 & 0.09 & 4.9 & 23.5 \\
P2000 & dash-dot-dot & 87067 & 2017 & 5120 & 768 & 2560 & 7.4 & 0.13 & 5.0 & 13.2 \\
P4000 & solid & 191333 & 4088 & 10240 & 1024 & 5120 & 7.5 & 0.19 & 5.0 & 3.31 \\
\hline
\end{tabular}

Table 1: List of parameters for turbulent channel flow cases. $R e_{b}=2 h u_{b} / \nu$ is the bulk Reynolds number, and $R e_{\tau}=h u_{\tau} / \nu$ is the friction Reynolds number. $N_{x}, N_{y}, N_{z}$ are the number of grid points in the streamwise, wall-normal, and spanwise directions. $\Delta x^{+}$, $\Delta z^{+}$are the grid spacings in the wall-parallel direction and $\Delta y_{w}^{+}$the minimum spacing in the wall-normal direction, in wall units. The simulation time $T$ is given in terms of eddy turnover times $h / u_{\tau}$. An error stretching function $y(\eta)=\operatorname{erf}[a(\eta-0.5)] / \operatorname{erf}(0.5 a)$, $a=4, \eta=[-1,1]$ has been used to cluster points in the wall-normal direction.

\begin{tabular}{cccc}
\hline & & & \\
\hline Scalar field & Symbol & $\operatorname{Pr}$ & Boundary conditions \\
\hline A & Square & 0.2 & Uniform forcing \\
B & Triangle & 0.71 & Uniform forcing \\
C & Diamond & 1 & Uniform forcing \\
D & Circle & 0.71 & Assigned difference \\
\hline
\end{tabular}

Table 2: Test conditions for passive scalar fields.

were observed for the low-wavenumber part of the spectra, whereas the scalar spectra were found to exhibit larger energy at high wavenumbers, especially in the outer region, where the scalar field was found to be less mixed than the velocity field.

The main motivation of the present study is to extend DNS of passive scalars to higher Reynolds numbers, specifically up to $R e_{\tau} \approx 4000$. It is known (Bernardini et al. 2014; Lee \& Moser 2015) that in this range of Reynolds numbers a near logarithmic layer starts to emerge in the mean velocity profile, hence asymptotic high-Reynolds-number effects are expected to manifest themselves which were not observed in previous studies.

\section{Computational setup}

We solve the Navier-Stokes momentum equations for a divergence-free velocity field

$$
\frac{\partial u_{j}}{\partial x_{j}}=0, \quad \frac{\partial u_{i}}{\partial t}+\frac{\partial u_{i} u_{j}}{\partial x_{j}}=-\frac{\partial p}{\partial x_{i}}+\frac{1}{R e} \frac{\partial^{2} u_{i}}{\partial x_{j} \partial x_{j}}+\Pi \delta_{i 1},
$$

where $\Pi$ is the driving force required to maintain a constant flow rate in the $x$ direction. The equations are discretized in a Cartesian coordinate system $(x, y, z$ denote the streamwise, wall-normal and spanwise directions) using staggered central second-order finite-difference approximations, to guarantee that kinetic energy is globally conserved in 
the limit of inviscid flow. Time advancement is carried out by means of a hybrid thirdorder low-storage Runge-Kutta algorithm coupled with the second-order Crank-Nicolson scheme combined in the fractional-step procedure, whereby the convective terms are treated explicitly and the diffusive terms are treated implicitly, limited to the wall-normal direction. The Poisson equation for the pressure field stemming from the incompressibility condition is efficiently solved through Fourier transform-based methods (Kim \& Moin 1985). A full description of the algorithm is provided in Orlandi (2000). The fluid equations are augmented with the transport equations for passive scalar fields with finite diffusion

$$
\frac{\partial \theta}{\partial t}+\frac{\partial \theta u_{j}}{\partial x_{j}}=\frac{1}{\operatorname{RePr}} \frac{\partial^{2} \theta}{\partial x_{j} \partial x_{j}}+Q
$$

where $\theta$ is the transported variable (temperature or concentration), $\operatorname{Pr}$ is either the Prandtl number or the Schmidt number, and $Q$ is a suitable source term. In the numerical discretization the transported variable is located at the cell center in such a way that the variance of $\theta$ is exactly preserved in the limit of inviscid flow. The source term is defined to be either zero in the case of walls with different values of $\theta$, or dynamically adjusted in such a way that the integral of $\theta$ over the channel is strictly constant in time, in analogy with the treatment of the driving force in the momentum equation. This approach is slightly different from that used by Kim \& Moin (1989), who also adopted a spatially uniform forcing, however with an a-priori selected source term $Q=1 / \operatorname{Pr}$. Our approach also differs from that used by Kawamura et al. (1998) and Abe et al. (2004b), who enforced a strictly constant wall flux in time by adding a spatially non-uniform source term. In our case, although the wall flux is not strictly constant in time, its value starts to fluctuate with excursions of no more than $1 \%$ with respect to the mean value, once a statistically steady state is achieved. For the sake of efficiency and accuracy (Kleiser \& Zang 1991; Bernardini et al. 2013) the computations are carried out in a reference frame in which the bulk velocity is zero. In addition to allowing a larger computational time step, this expedient minimizes the dispersion errors associated with finite-difference discretization.

Details on the computational mesh and on the parameters used for DNS of the fluid phase are provided in table 1 . The set-up is very similar to our previous study (Bernardini et al. 2014), with the main difference of slightly improved resolution in all coordinate directions. The DNS have been carried out in a $\left(L_{x} \times L_{y} \times L_{z}\right)=(6 \pi h \times 2 h \times 2 \pi h)$ computational box, which is sufficiently long to accommodate the largest outer-layer flow structures. Points are clustered towards the walls according to an error function mapping, to have the first point at $\Delta y_{w}^{+} \approx 0.1$, and the maximum spacing in terms of local Kolmogorov units is $(\Delta y / \eta)_{\max } \approx 2.4$ for all flow cases. The resolution in the wall-parallel directions in terms of Kolmogorov units is $\Delta x / \eta \lesssim 5.3, \Delta z / \eta \lesssim 3.6$ throughout. Since the maximum Prandtl number is unity Batchelor units (Batchelor 1959) are larger than or equal to Kolmogorov units, hence the resolution for the scalar fields is no worse than for the velocity field. The adequacy of the mesh resolution has been checked by monitoring the streamwise and spanwise passive scalar spectral densities (not shown here). The absence of any energy pile-up and the exponential decay of the high-wavenumber-end of the spectra is a strong hint that the sharp gradients of passive scalar fields are correctly resolved. A grid sensitivity study has also been performed for the P550 flow case, which has shown that doubling the mesh points in the wall-parallel directions yields a change of less than $1 \%$ in the peak scalar variance. The adequacy of the time interval for the statistical analysis was also tested for the most critical $\mathrm{P} 4000$ flow case by comparing the mean and the variance of the passive scalar field, collected in quarters of the full window. 
(a)

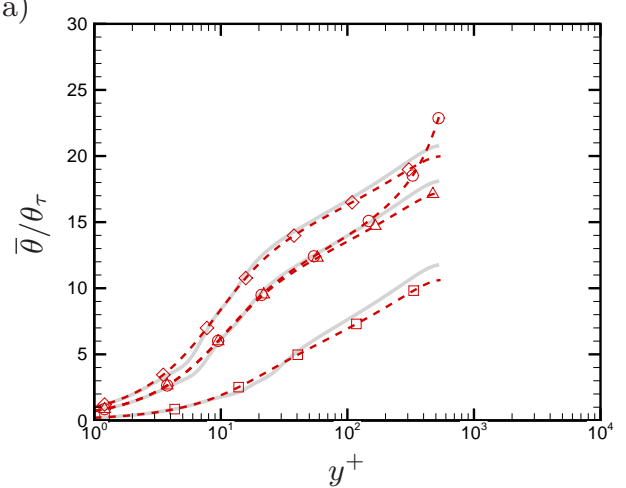

(c)

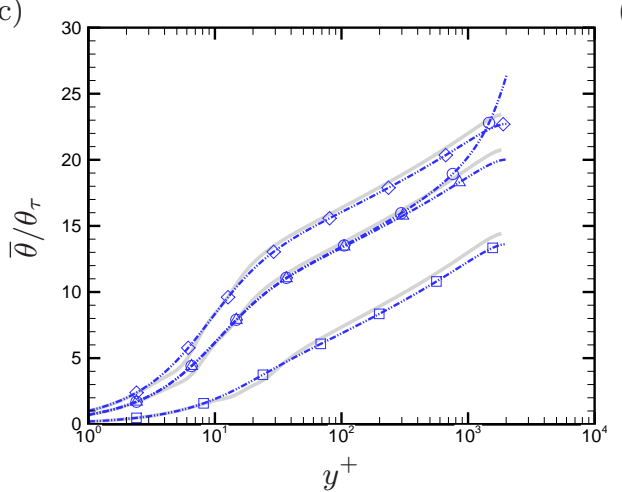

(b)

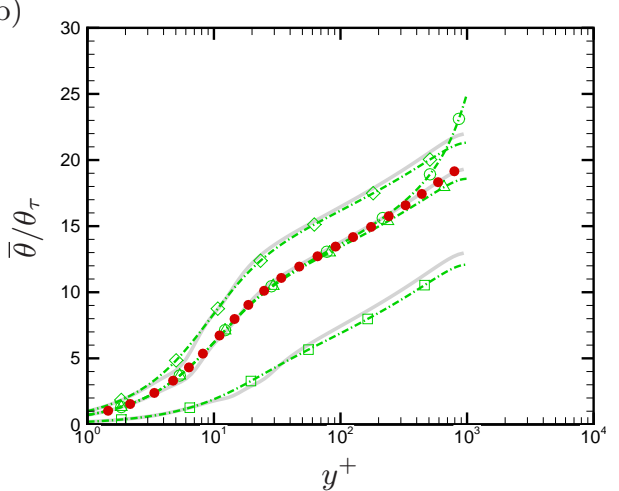

(d)

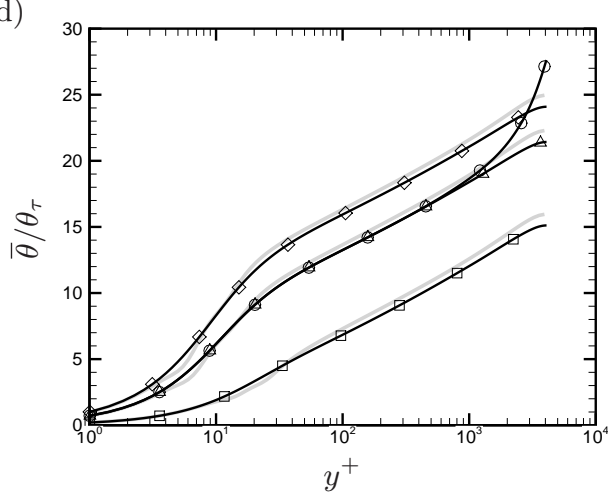

Figure 1: Mean scalar profiles for flow cases P550 (a), P1000 (b), P2000 (c), P4000 (d). The data are compared with the fits of Kader (1981) (thick grey lines) and with DNS data of Abe et al. (2004b) at $R e_{\tau}=1020$ (solid symbols in panel b). Refer to tables 1,2 for nomenclature of the DNS data.

The results of this exercise (not shown) indicate scatter of about $1 \%$, confined to the outer wall layer. Four different conditions for the passive scalar fields are considered (see table 2) for each $R e_{\tau}$, including three simulations with uniform forcing and $\theta=0$ at the two walls, and one with zero forcing and assigned difference between the two walls $(\theta= \pm 1)$. Of special interest is the case $\operatorname{Pr}=1$ (scalar field $\mathrm{C}$ ), which obeys to the same equations as the streamwise velocity field, in the absence of nonlinear feedback effects and in the absence of redistribution to the other velocity components through the pressure-strain term (Pope 2000).

\section{Results}

\subsection{Mean and r.m.s. values}

The mean scalar distributions are given in figure 1 in wall units, where the friction temperature is defined as

$$
\theta_{\tau}=\left.\frac{\alpha}{u_{\tau}} \frac{\mathrm{d} \bar{\theta}}{\mathrm{d} y}\right|_{w}
$$

$\alpha$ being the scalar diffusivity. Semi-empirical fits for the mean scalar profiles in channel flows with constant heating were given by Kader (1981) based on available experimental data, under the main assumption that the overlap layer exhibits logarithmic variation of 
the type

$$
\frac{\bar{\theta}-\bar{\theta}_{w}}{\theta_{\tau}}=\frac{1}{k_{\theta}} \log y^{+}+C_{\theta}(\operatorname{Pr}),
$$

where $k_{\theta}$ is the Karman constant for the scalar field, and the additive constant is an empirically determined function of the Prandtl number. The curve fits of Kader (1981) with the original set of constants (including $k_{\theta}=0.47$ ) are compared with the DNS data in figure 1. All passive scalar profiles visually exhibit a near-logarithmic layer which is widening with $R e_{\tau}$ and, as well established, the effect of Prandtl number increase is the increase of the additive constant in the logarithmic law, the slope of the graphs being visually the same. The scalar field $\mathrm{D}$ with assigned difference at the two walls has a different behavior than the others, with mean profiles which start to deviate from the corresponding forced case $\mathrm{B}$ at $y / h \approx 0.2$ and sloping toward the channel centerline where the mean gradient in not zero, similar to the mean velocity profile of Couette flow. This behavior led (Kawamura et al. 2000) to conclude, based on low-Re simulations, that the scalar Karman constant should be different between the forced and assigned temperature case. However, based on the present high-Re data we are led to conclude that the mean scalar profile in the logarithmic layer is very little affected by the type of forcing. Overall good agreement with Kader's correlation is found, with larger deviations at low Prandtl number. Kader's formula typically overshoots the numerical values, with deviations up to $4 \%$ in the log layer. Reasons for this difference may reside in the fact that the wall heat flux is not precisely constant in the DNS. The DNS data of Abe et al. (2004b) at $R e_{\tau}=1020, \operatorname{Pr}=0.71$ are also shown for comparison in panel (b). While the logarithmic part of the profile is very similar to ours, a fuller scalar profile is found in their DNS which better agrees with Kader's fit. Additional simulations has been carried out In order to clarify this issue we have carried out additional DNS using the same computational set-up as Abe et al. (2004b) in terms of box size and/or scalar forcing scheme. Nearly identical results as Abe et al. (2004b) are obtained when the same forcing is used, which suggests that the forcing has an impact on the computed statistics, at least as far as the core part of the channel is concerned.

The inner-scaled profiles exhibit clear Prandtl number sensitivity of the scalar fields, owing to different thickness of the conductive sublayer. To highlight outer scaling, the velocity and scalar profiles are shown in defect form in figure 2 . The figure suggests close universality of the profiles with respect to both Reynolds and Prandtl number, in line with theoretical expectations (Monin \& Yaglom 1971). In the overlap layer, the scalar profiles are nearly logarithmic, with

$$
\frac{\bar{\theta}_{\mathrm{CL}}-\bar{\theta}}{\theta_{\tau}}=-\frac{1}{k_{\theta}} \log \eta+B_{\theta},
$$

where $\eta=y / h$, and $\bar{\theta}_{\mathrm{CL}}$ is the mean centerline value. Fitting the DNS data in defect representation is easier than for the inner-layer representation, and yields an approximate value for the Karman constant for the scalar fields of $k_{\theta} \approx 0.46$, and an additive constant $B_{\theta} \approx 0.18$. For comparison, fitting the defect velocity profile (see figure $2 \mathrm{a}$ ) yields $k \approx$ $0.41, B \approx 0.46$, hence the wake strength is found to be greater in the velocity field than in the scalar fields.

The shape of the scalar profiles in the core layer of channels have been the subject of several conjectures in the past (Monin \& Yaglom 1971; Kader 1981; Cebeci \& Bradshaw 1984), which typically led to empirical polynomial fits of the scalar profiles from experimental data. It has been recently shown (Pirozzoli 2014; Orlandi et al. 2015) that the crude assumption of uniform eddy viscosity is rather accurate in predicting the mean ve- 
(a)

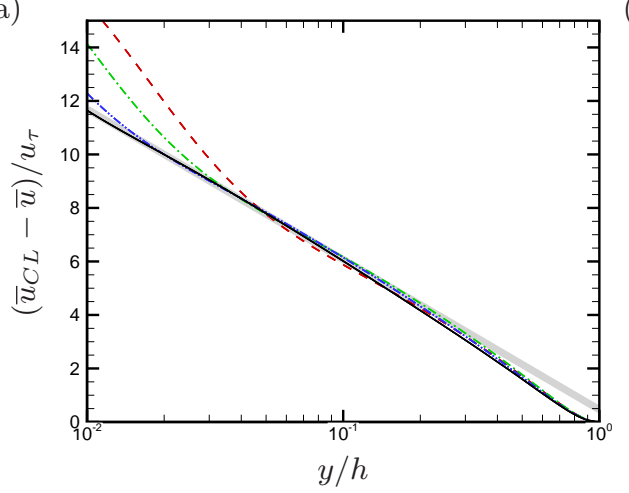

(c)

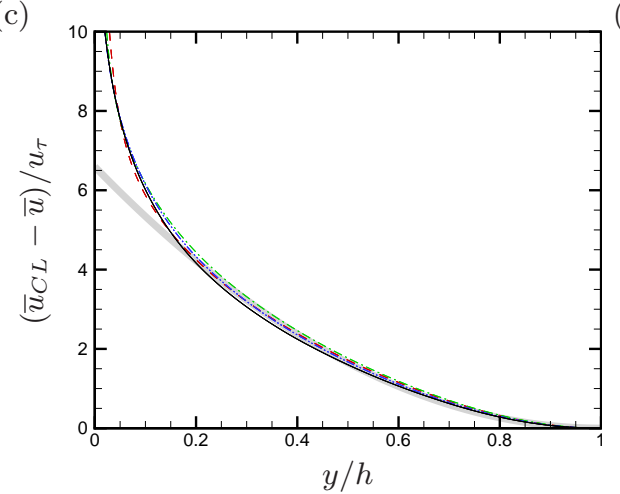

(b)

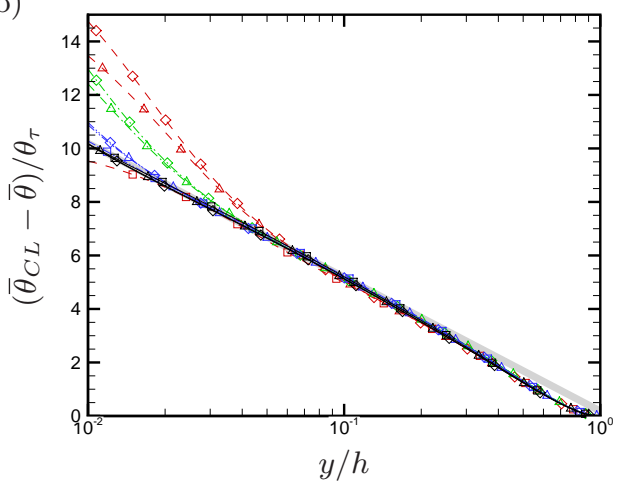

(d)

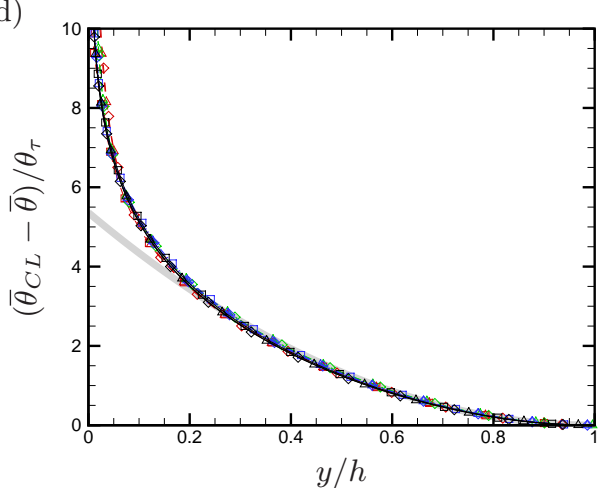

Figure 2: Mean velocity (a,c) and scalar (b,d) profiles in defect form for scalar fields A-C. Logarithmic fits of the overlap profiles are shown in panels (a,b), and parabolic fits of the core profiles are shown in panels $(\mathrm{c}, \mathrm{d})$ with thick grey lines. Refer to tables 1,2 for nomenclature of the DNS data.

locity profiles for all canonical wall-bounded flows, including Poiseuille flow. Specifically, under the assumption that the relevant outer eddy velocity scale is $u_{\tau}$ and the typical size is $h$ one has

$$
\nu_{t}=c_{\mu} u_{\tau} h
$$

where $c_{\mu}$ is a suitable constant, whence the parabolic core mean velocity law follows

$$
u_{\mathrm{CL}}^{+}-u^{+}=\frac{1}{2 c_{\mu}}(1-\eta)^{2} .
$$

We argue that a uniform eddy diffusivity assumption may also apply to passive scalar fields, under the ansatz

$$
\alpha_{t}=c_{\alpha} u_{\tau} h
$$

which yields the parabolic core scalar law

$$
\theta_{\mathrm{CL}}^{+}-\theta^{+}=\frac{1}{2 c_{\alpha}}(1-\eta)^{2} .
$$

Fitting equation (3.7) to the DNS data in figure 2 yields $c_{\mu} \approx 0.076, c_{\alpha} \approx 0.093$, the relevant turbulent Prandtl number being $P r_{t}=\nu_{t} / \alpha_{t} \approx 0.81$. The accuracy of the fit is apparently very good down to $\eta \approx 0.2$, regardless of the Reynolds and Prandtl number.

Although the inner- and outer-scaled scalar profiles appear to exhibit near-logarithmic 
(a)

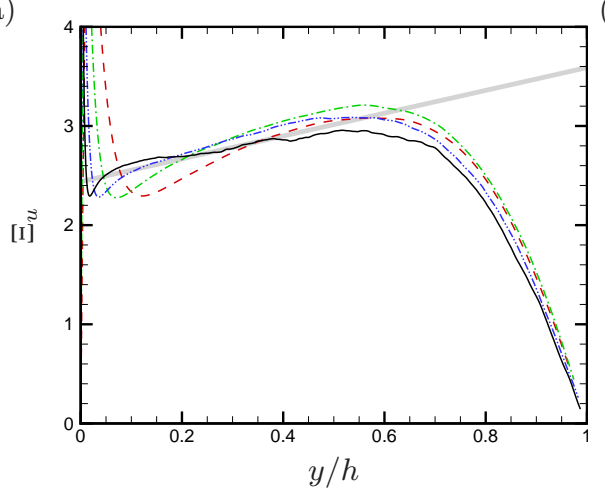

(b)

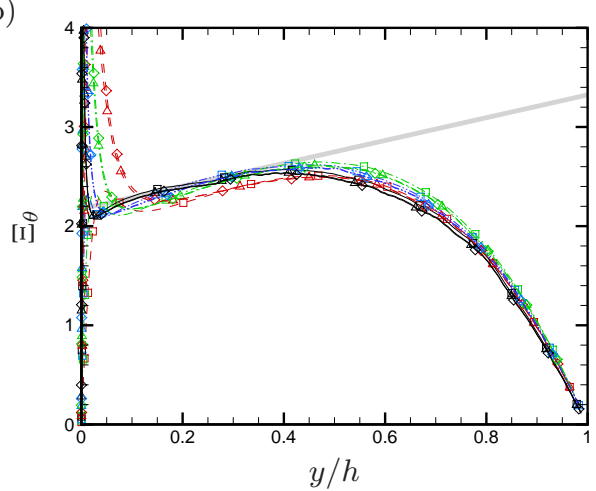

Figure 3: Diagnostic function for mean velocity (a) and for scalar fields A-C (b). The thick grey lines correspond to equation (3.8) with $k=0.41, \gamma=1.1, \beta=0$ (a), and $k_{\theta}=0.46, \gamma_{\theta}=1.15, \beta_{\theta}=0$ (b). Refer to tables 1,2 for nomenclature of the DNS data.

layers, and the log-layer approximation is in fact satisfactory for most practical purposes, a more into-depth analysis shows that the situation is probably more intricate (Pirozzoli 2014). In figure 3 we show the log-law diagnostic function, $\Xi_{u}=y^{+} \mathrm{d} u^{+} / \mathrm{d} y^{+}, \Xi_{\theta}=$ $y^{+} \mathrm{d} \theta^{+} / \mathrm{d} y^{+}$, for the velocity and the scalar fields. Clearly, constancy of $\Xi$ would indicate the presence of a genuine log layer. As noticed by Bernardini et al. (2014), no clear plateau is found in $\Xi_{u}$, neither for the velocity nor for the scalar field. However, there is rather clear evidence for the presence of a range with nearly linear variation of $\Xi_{u}$ with the wall distance, whose slope (in inner units) decreases with $R e_{\tau}$, and which is predicted by some refined overlap theories (Afzal \& Yajnik 1973). According to such generalized $\log$ layer formulations,

$$
\Xi=\frac{1}{k}+\gamma \eta+\frac{\beta}{R e_{\tau}}
$$

where (Bernardini et al. 2014), $k \approx 0.41, \gamma \approx 1.1, \beta \approx 180$ for the streamwise velocity field. Fitting the mean scalar profiles in figure $3(\mathrm{~b})$ in the range $100 / R e_{\tau} \leqslant \eta \leqslant 0.5$ yields $k_{\theta} \approx 0.46, \gamma_{\theta} \approx 1.15 \approx \gamma$, with very weak effect of the molecular Prandtl number. It should be noted that the presence of a generalized logarithmic layer does not prevent the onset of a narrow genuine logarithmic layer at higher Reynolds, which however should be confined to a narrower range of wall distances (Lee \& Moser 2015).

The variances of the streamwise velocity and scalar fluctuations are shown in figure 4 in inner and outer scaling. The inner-scaled scalar profiles (panel (b)) are strongly affected by the molecular conductivity, being an increasing function of $\operatorname{Pr}$. As expected based on the formal similarity of the governing equations, the shape of the scalar variance profiles at $\operatorname{Pr}=1$ is similar to the streamwise velocity variance, with the main exception of a higher value of the near-wall peak. Of course, the scalar variance in flow case D has a very different behavior in the outer layer, where production does not drop to zero (Kawamura et al. 2000), and a second peak emerges which is larger than the nearwall one, and also increasing with $R e_{\tau}$. Comparison with previous DNS by Abe et al. $(2004 b)$ is quite favourable, whereas substantial differences are found with respect to available experimental data of Subramanian \& Antonia (1981), not shown. The velocity and scalar variances are shown in defect form in panels (c), (d). Collapse in the defect representation is but fair for the velocity field, whereas more convincing universality over the Re and $\operatorname{Pr}$ range is found for the scalar variances. The odd behavior of the 
(a)
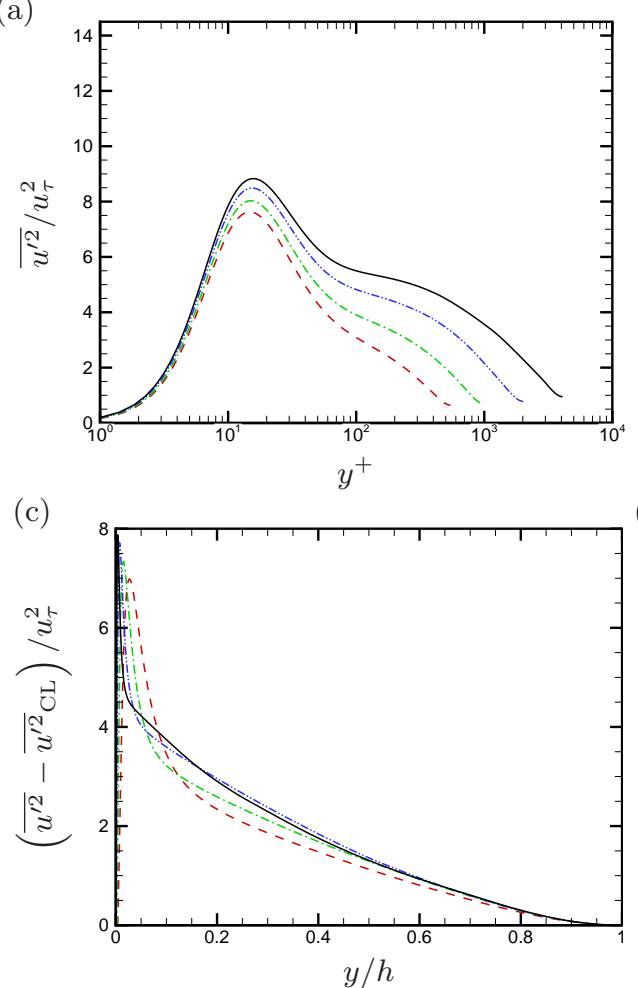

(b)

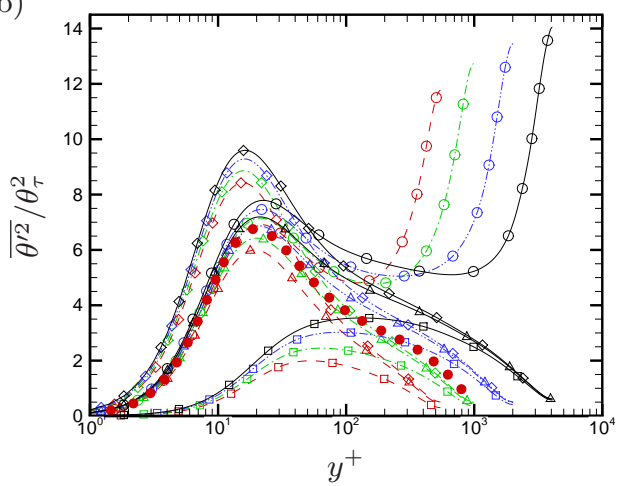

(d)

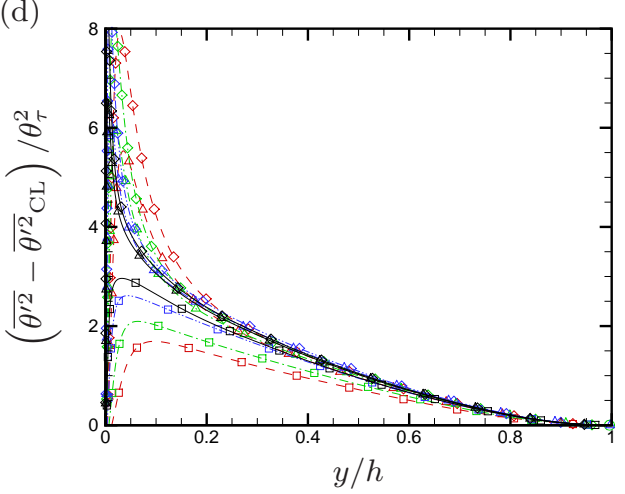

Figure 4: Streamwise velocity variance $(\mathrm{a}, \mathrm{c})$ and scalar variance $(\mathrm{b}, \mathrm{d})$ as a function of the wall distance in inner units $(a, b)$ and outer units (c,d). In panel (b) the solid circles indicate DNS data of Abe et al. (2004b). Refer to tables 1,2 for nomenclature of the DNS data.

streamwise velocity variance is at the root of alternative (mixed) scalings which have been proposed over the years (DeGraaff \& Eaton 2000; del Álamo et al. 2004), and it is probably related to the difficulty to identify a logarithmic layer as requested by the attached eddy hypothesis (Townsend 1976; Perry \& Marusic 1995; Orlandi et al. 2015).

The peaks of the scalar and streamwise velocity variances are shown in figure 5 as a function of the Reynolds number. Attached-eddy arguments support logarithmic increase of the inner peak of the streamwise velocity variance with $R e_{\tau}$ because of the increasing effect of overlying attached eddies (Townsend 1976; Hutchins et al. 2009). The present data suggest that the same behavior also applies to passive scalar fields. Specifically, we find that the growth rate is approximately the same at all $\mathrm{Pr}$, with a strongly increasing additive constant. The case of assigned wall difference shows moderate increase in the near-wall peak for given $P r$, owing to energy leakage from stronger eddies forming in the channel core (also see the later spectral maps). As previously noticed, scalar field D also exhibits a prominent peak at the channel centerline, whose amplitude (solid circles in figure 5) shows a similar growth rate with $R e_{\tau}$ as the near-wall peaks.

Similarities between passive scalar and streamwise velocity statistics are more closely scrutinized in figure 6 , where we compare the variances and the vertical turbulent fluxes for $\operatorname{Pr}=1$ (scalar field C). As previously noticed, the scalar variances attain a higher peak in the near-wall region, whereas they tend to form log-like layers for $100 / R e_{\tau} \leqslant \eta \leqslant 0.2$. In this region the streamwise velocity variance is higher and it tends to form a 'bump' 


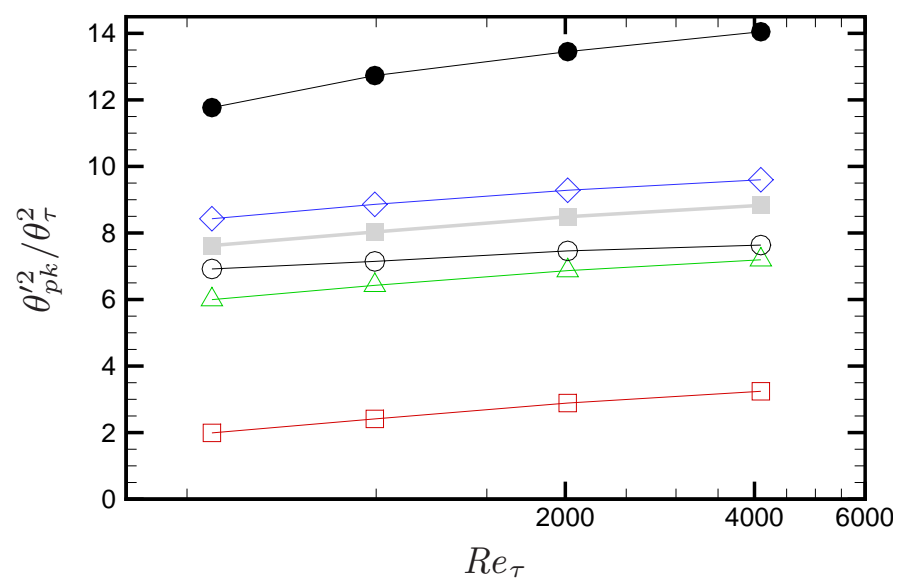

Figure 5: Peak scalar variances at various $\operatorname{Pr}$ (see table 2 for nomenclature of symbols) as a function of $R e_{\tau}$, compared with the peak streamwise velocity variance (solid squares). The solid circles denote the centerline peak of the scalar variance for field D.
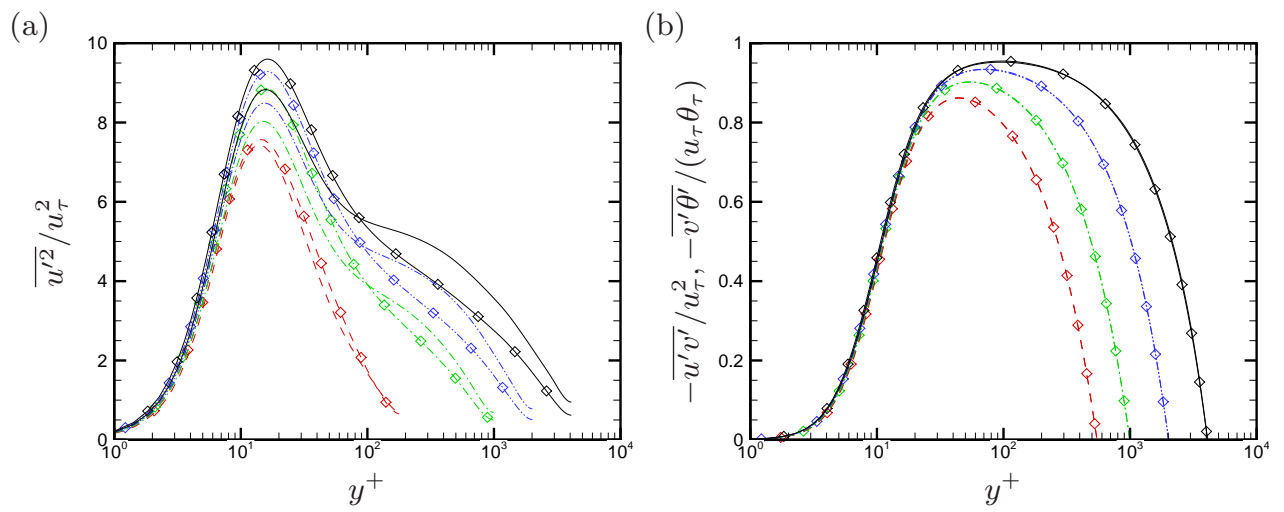

Figure 6: Variances (a) and vertical turbulent fluxes (b) for streamwise velocity field (lines) and passive scalar field C (lines+symbols). Refer to tables 1,2 for nomenclature.

which precludes the observation of a logarithmic layer, although no evidence for an outer peak is found at the Reynolds numbers under scrutiny. On the other hand, the vertical turbulent fluxes, shown in figure 6(b) are (visually) very nearly indistinguishable, hence suggesting that the lift-up mechanism which is responsible for the $u-v$ and $v-\theta$ correlations is very nearly linear in nature. At the same time, it must be stated that small differences in the vertical turbulent fluxes are at the core of observable differences in the mean profiles, which were clear in figure 1.

\subsection{Energetics}

Differences in the behavior of scalar and streamwise velocity variances can be further elaborated by monitoring the respective budget equations, reported below (Pope 2000)

$$
0=-\frac{\mathrm{d} \overline{v^{\prime} u^{\prime 2} / 2}}{\mathrm{~d} y}+\nu \frac{\mathrm{d}^{2} \overline{u^{\prime 2} / 2}}{\mathrm{~d} y^{2}} \underbrace{-\overline{u^{\prime} v^{\prime}} \frac{\mathrm{d} \bar{u}}{\mathrm{~d} y}}_{\mathcal{P}_{11}}-\frac{\mathrm{d} \overline{p^{\prime} v^{\prime}}}{\mathrm{d} y}+\underbrace{\overline{p^{\prime} \frac{\partial u^{\prime}}{\partial x}}}_{\mathcal{R}_{11}}-\underbrace{\nu \overline{\frac{\partial u^{\prime}}{\partial x_{k}} \frac{\partial u^{\prime}}{\partial x_{k}}}}_{\varepsilon_{11}},
$$


(a)

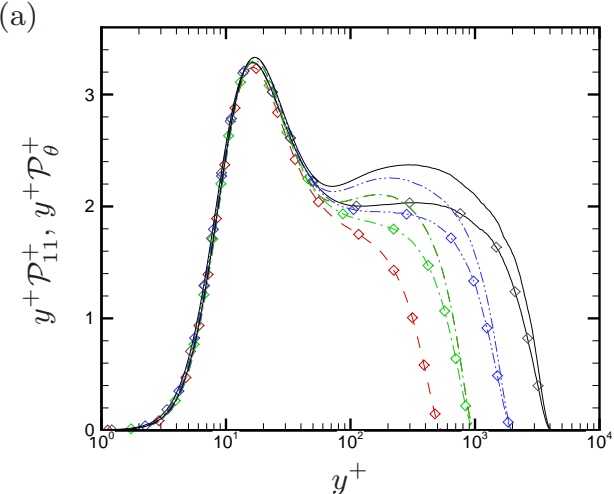

(b)

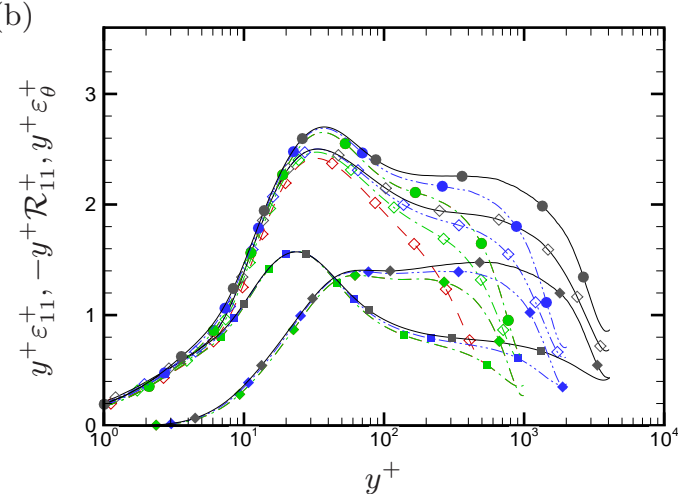

Figure 7: Pre-multiplied production (a) and dissipation rate (b) of streamwise velocity field (lines) and passive scalar field C (lines+open symbols). The solid symbols in panel (b) denote the contributions of viscous dissipation $\varepsilon_{11}$ (squares) and pressure $\mathcal{R}_{11}$ (diamonds), and their sum (circles). Refer to table 1 for the line style.

$$
0=-\frac{\mathrm{d} \overline{v^{\prime} \theta^{\prime 2} / 2}}{\mathrm{~d} y}+\alpha \frac{\mathrm{d}^{2} \overline{\theta^{\prime 2} / 2}}{\mathrm{~d} y^{2}} \underbrace{-\overline{\theta^{\prime} v^{\prime}} \frac{\partial \bar{\theta}}{\partial y}}_{\mathcal{P}_{\theta}}-\underbrace{\alpha \frac{\overline{\partial \theta^{\prime}}}{\partial x_{k}} \frac{\partial \theta^{\prime}}{\partial x_{k}}}_{\varepsilon_{\theta}} .
$$

In figure 7 we show the source terms in equations (3.9), (3.10) associated with production $\left(\mathcal{P}_{11}\right.$ and $\mathcal{P}_{\theta}$, respectively), viscous dissipation $\left(\varepsilon_{11}\right.$ and $\left.\varepsilon_{\theta}\right)$, and pressure-strain correlation $\left(\mathcal{R}_{11}\right.$, absent in the scalar variance equation). It must be noted that, in order to more closely compare small terms (all scaling as $1 / y$ ), the various quantities are reported in pre-multiplied form in semi-log representation, so that equal areas underneath the curves correspond to equal integral contributions. The near-wall region (say $y^{+} \lesssim 100$ ) is characterized by nearly equal values of velocity and scalar production. On the other hand, similarity of velocity and scalar dissipation is confined to the viscous sublayer, consistent with the analogy noticed by Abe \& Antonia (2009). Further away from the wall the dissipation rates become very different, the velocity dissipation being much less than the scalar dissipation. However, this difference is more than compensated by the pressure term in the streamwise momentum equation, which should hence be regarded as responsible for the lower near-wall peak of the velocity variance as compared to the scalar variance. In the outer wall layer streamwise momentum production significantly exceeds scalar production, thus making up for the previously noticed outer bump in the streamwise velocity variance. Consistent with greater production, the total velocity dissipation is also found to exceed scalar dissipation, mostly because of the pressure term, whose pre-multiplied distribution tends to form an outer peak.

The effect of the various source terms in the variance budgets can be appreciated by inspecting the global energy balance equations, which are easily derived from the streamwise momentum and scalar transport equations

$$
\underbrace{h \Pi u_{b}}_{W}=\underbrace{\int_{0}^{h} \varepsilon_{11} \mathrm{~d} y}_{V_{11}}+\underbrace{\int_{0}^{h}-\mathcal{R}_{11} \mathrm{~d} y}_{T}+\underbrace{\nu \int_{0}^{h}(\mathrm{~d} \bar{u} / \mathrm{d} y)^{2} \mathrm{~d} y}_{\bar{V}_{11}}
$$


12

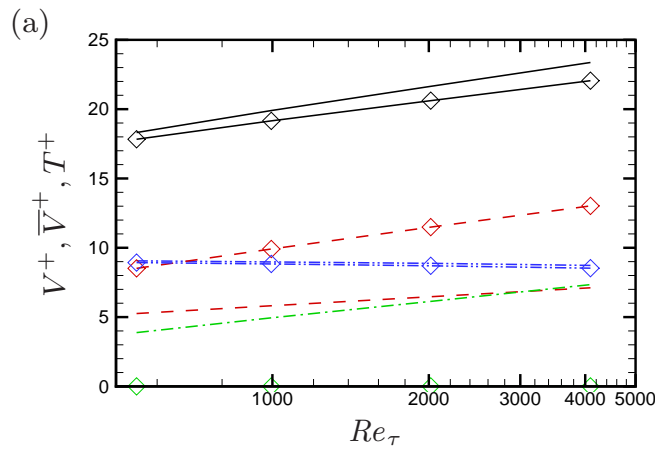

S. Pirozzoli, M. Bernardini, P. Orlandi

(b)

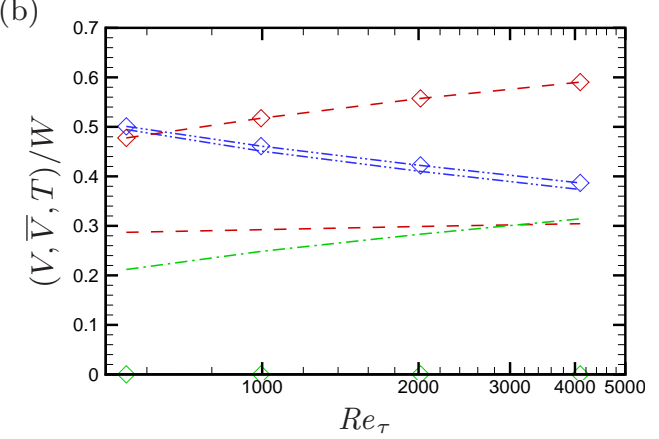

Figure 8: Global energy balance of streamwise velocity (lines) and scalar field C (lines and symbols) as a function of Reynolds number, expressed in wall units (a) and as a fraction of the total power expenditure (b). Solid lines: total power expenditure $(W)$; dashed lines: turbulent dissipation $(V)$; dash-dot-dotted lines: mean flow dissipation $(\bar{V})$; dash-dotted lines: pressure transfer $(T)$.

$$
\underbrace{h Q \theta^{*}}_{W_{\theta}}=\underbrace{\int_{0}^{h} \varepsilon_{\theta} \mathrm{d} y}_{V_{\theta}}+\underbrace{\alpha \int_{0}^{h}(\mathrm{~d} \bar{\theta} / \mathrm{d} y)^{2} \mathrm{~d} y}_{\bar{V}_{\theta}},
$$

where $\theta^{*}=1 / h \int_{0}^{h} \bar{\theta} \mathrm{d} y$ is the mean value of $\theta$ across the channel, which states that the power spent to force the momentum and the scalar transport equations is partly dissipated by the mean flow (the $\bar{V}$ terms) and by turbulent motions (the $V$ terms), and partly to produce the wall-normal and spanwise velocity components (the $T$ redistributive term). The budget terms of equations (3.11) and (3.12) are shown in figure 8, in wall units (panel (a)) and as a fractional contribution to the overall power expenditure (b). As expected, the power expenditure $(W)$ in wall units (which reduces to $W^{+}=u_{b}^{+}=$ $\sqrt{2 / C_{f}} \sim \log R e_{\tau}$ in the streamwise momentum equation) is increasing logarithmically with $R e_{\tau}$, both for the velocity and for the scalar field, although slightly faster growth is found for the former. In the scalar field the growth is clearly associated with growth of the turbulent dissipation term, whereas mean flow dissipation is very weakly decreasing with $R e_{\tau}$. In the streamwise velocity field mean flow dissipation is again nearly constant, but both turbulent dissipation and pressure redistribution are increasing with $R e_{\tau}$. As clearer in panel (b), the latter term is increasing faster, and it is expected to dominate in the asymptotic high-Re regime, if the slow logarithmic trends here observed are confirmed.

The production excess over the total dissipation is displayed in figure 9 . It appears that a production excess of up to about about $5 \%$ is present from $y^{+} \approx 200$ to $y / h \approx 0.5$ in the velocity field and in the scalar fields with uniform forcing. On the other hand, scalar field $\mathrm{D}$ with assigned wall difference has excess production in the core of the channel, say $y / h \gtrsim 0.6$, where the large eddies reside. It is noteworthy that, in outer representation, the production excess curves attain a common outer envelope which, if extrapolated would probably lead to a finite value in the infinite Reynolds number limit. This outer-layer production excess has been previously linked to the presence of large energy-containing eddies in Poiseuille flow (Bernardini et al. 2014), and it is more apparent in Couette flow (Pirozzoli et al. 2014). This is confirmed by the flow visualizations of figure 10, where we report instantaneous cross-stream contours of $u^{\prime}$ and $\theta^{\prime}$ (for scalar fields C and D), for flow case P4000, taken at the same time instant. The velocity field and the scalar field in the forced case away from walls is organized into 'towering' eddies which 
(a)

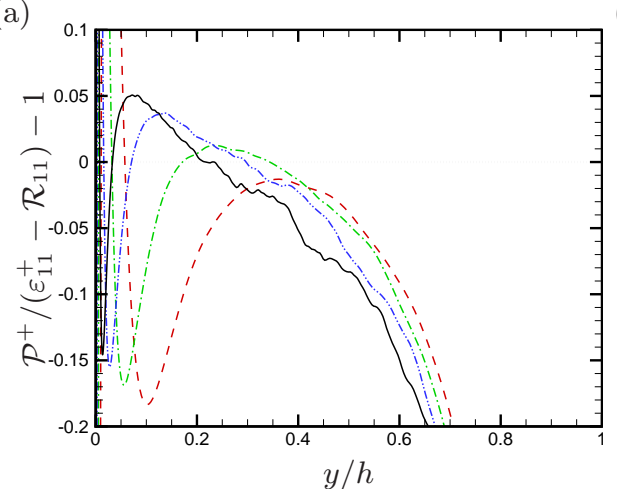

(c)

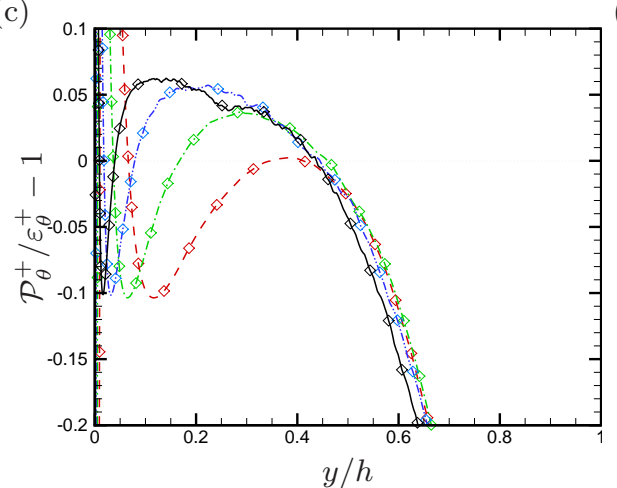

(b)

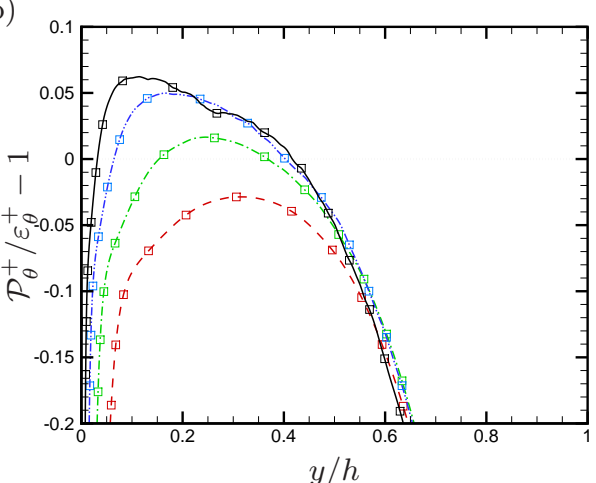

(d)

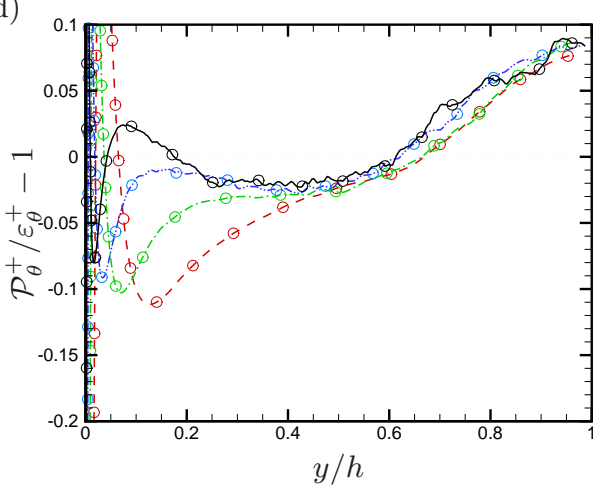

Figure 9: Outer-scaled production-to-dissipation excess for streamwise velocity field (a), and scalar fields A (b), C (c), D (d). Refer to tables 1,2 for nomenclature of the DNS data.

are attached to the wall, and which exhibit spanwise growth with the wall distance. Particularly striking is the similarity between the streamwise velocity fluctuation field and the passive scalar field $\mathrm{C}$, which clearly indicates that eddies supporting momentum fluctuations also carry the scalar field. In this respect it should also be noted that the interfaces between neighboring eddies are visually sharper in the scalar field, whereas they are more 'blurred' in the velocity field, which is a likely consequence of its active behavior. Similar observations at lower Reynolds number were reported by Antonia et al. (2009). Also striking is the similarity between the two scalar fields in the lower part of the channel (say $y / h \lesssim 0.5$ ) where the correlation coefficient (not shown) is always larger than 0.8. However, a different organization is recovered in the channel core, where scalar field D exhibits very large eddies, reminiscent of the momentum eddies in Couette flow (Pirozzoli et al. 2014).

This scenario is quantitatively confirmed by the spectral maps shown in figure 11. In order to highlight scale changes, in the figure we show the spanwise spectral densities of the streamwise velocity and of the scalar fields, normalized by the respective variance, by defining

$$
\hat{E}_{\varphi}\left(k_{z}\right)=E_{\varphi}\left(k_{z}\right) / \overline{\varphi^{\prime 2}} .
$$

The maps clearly bring out a two-scale organization of the flow field, with a near-wall peak associated with the wall regeneration cycle (Jiménez \& Pinelli 1999), and an outer peak associated with outer-layer large-scale motions (Hutchins \& Marusic 2007). The 
(a)

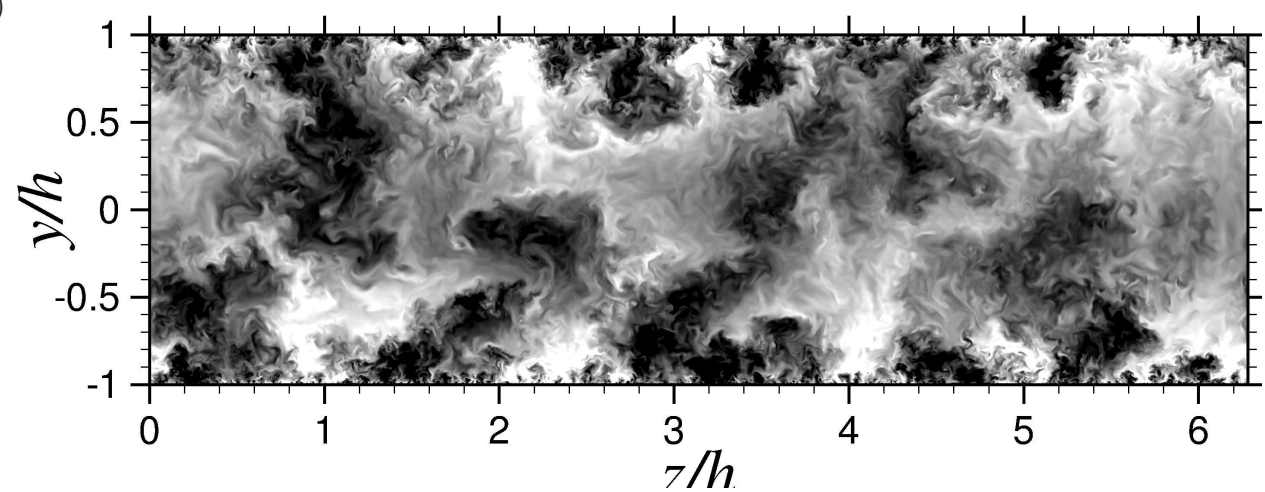

(b)

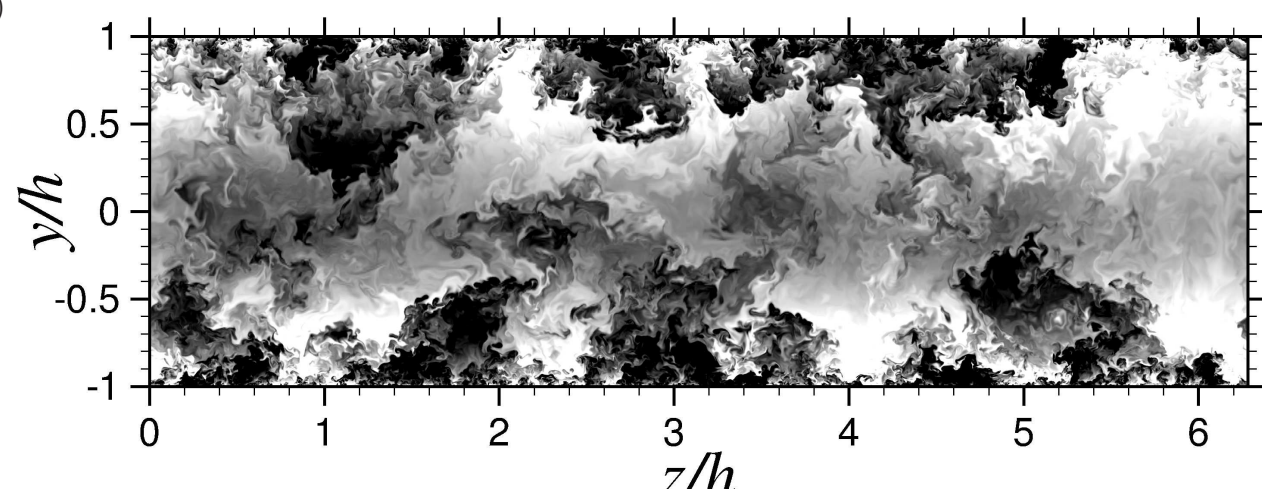

(c)

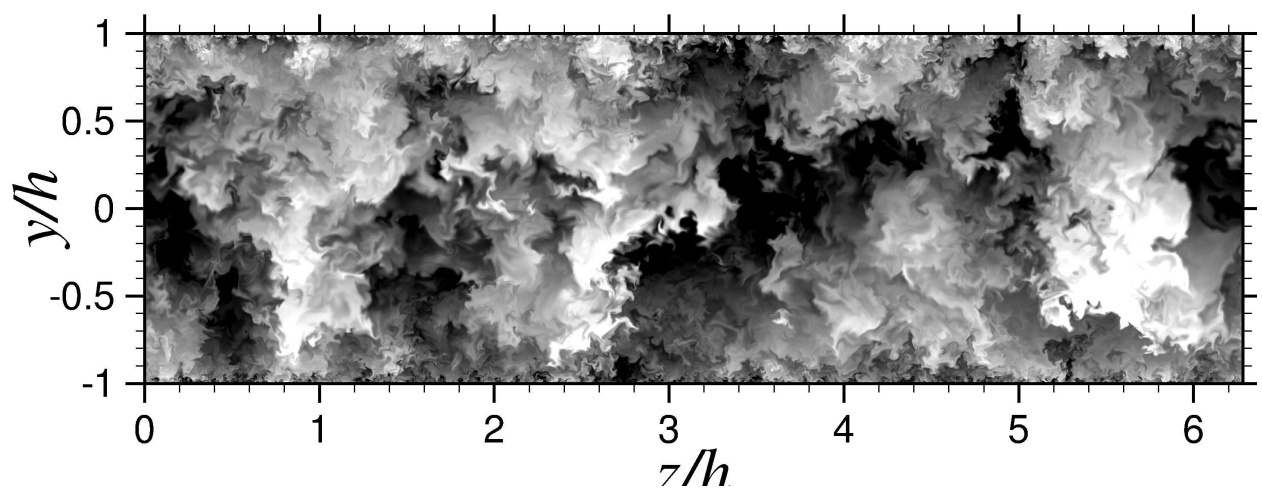

Figure 10: Instantaneous cross-stream visualizations of $u^{\prime}$ (a), and $\theta^{\prime}$ for scalar field C (b) and D (c), for flow case P4000. Panel (a): from $-3 u_{\tau}$ to $3 u_{\tau}$; panel (b): from $-2 \theta_{\tau}$ to $2 \theta_{\tau}$; from $-4 \theta_{\tau}$ to $4 \theta_{\tau}$. Sixty-four contour levels are shown, in shades from black to white.

latter peak is found to be centered at $y / h \approx 0.3$, and to correspond to eddies with typical wavelength $\lambda_{z} / h \approx 1$, consistent with the findings of Abe et al. (2004a). Secondary peaks corresponding to harmonics of this fundamental wavelength are observed at the higher Reynolds numbers, suggesting that the typical outer modes are not purely sinusoidal with respect to the spanwise direction. Notably, the same organization is recovered in 
P1000

(a)

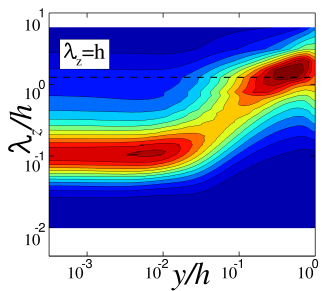

(d)

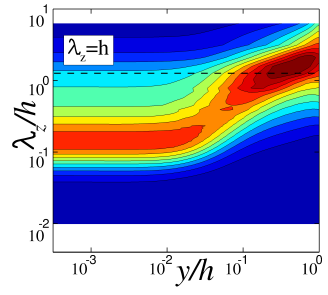

(g)

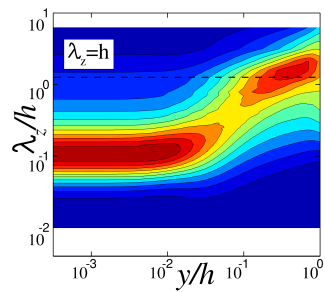

(j)

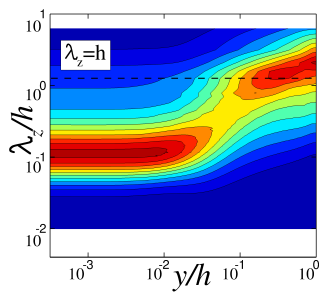

(b)

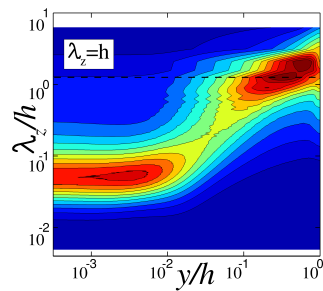

(e)

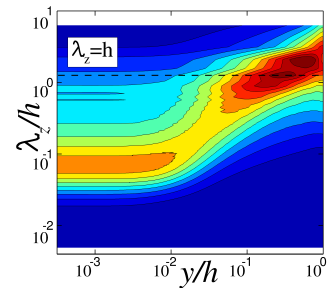

(h)

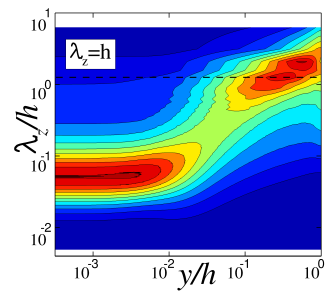

(k)

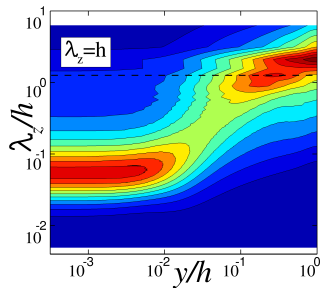

P4000

(c)

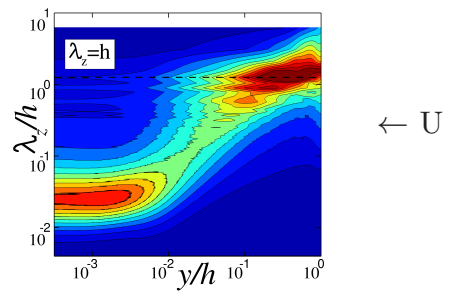

(f)

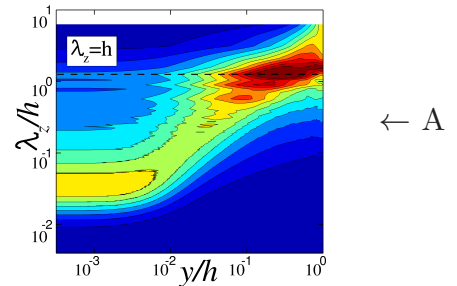

(i)

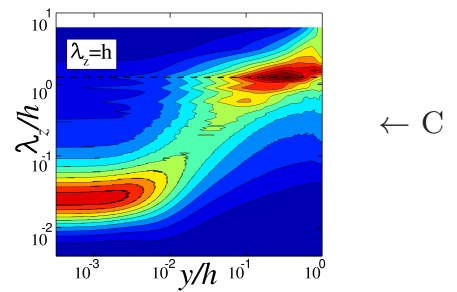

(l)

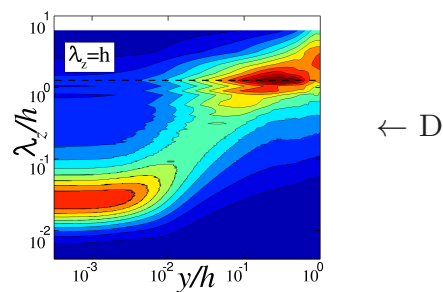

Figure 11: Variation of pre-multiplied, normalized spanwise spectral densities with wall distance: (a-c) spectra of $u\left(k_{z} \hat{E}_{u}\right)$ for flow cases P1000 (a), P2000 (b), P4000 (c); (d-l) spectra of $\theta\left(k_{z} \hat{E}_{\theta}\right)$ for flow cases P1000 (d,g,j), P2000 (e,h,j), P4000 (f,i,l), for the scalar fields A (d-f), C (g-i), D (j-l). Contour levels from 0 to 0.5 are shown, in intervals of 0.04 .

the scalar fields. While the near-wall peak sensitively depends in magnitude (but not position and wavelength) on the Prandtl number, being inhibited by diffusion at low $\operatorname{Pr}$, the outer peak is quite robust and universal with respect to both $R e$ and $\operatorname{Pr}$. The main effect of passive scalar forcing is the presence of extra energy near the channel centerline, associated with the large core eddies of figure 10c.

The structure of the flow field and the statistical association between velocity and scalar 
(a)

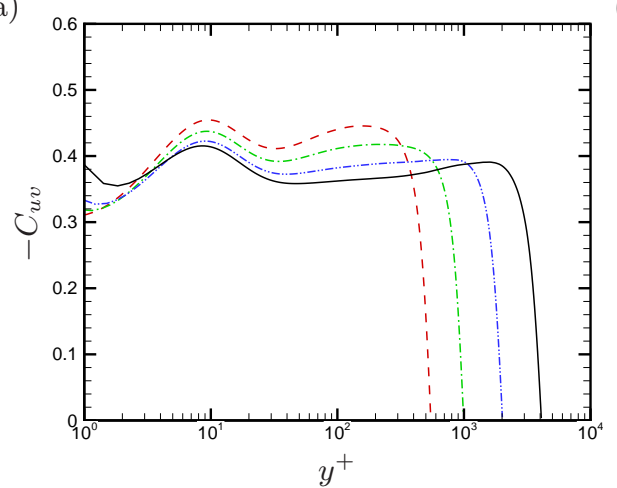

(b)

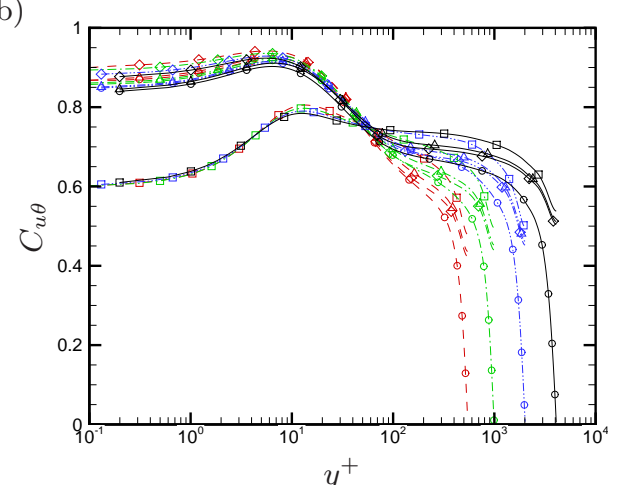

(c)

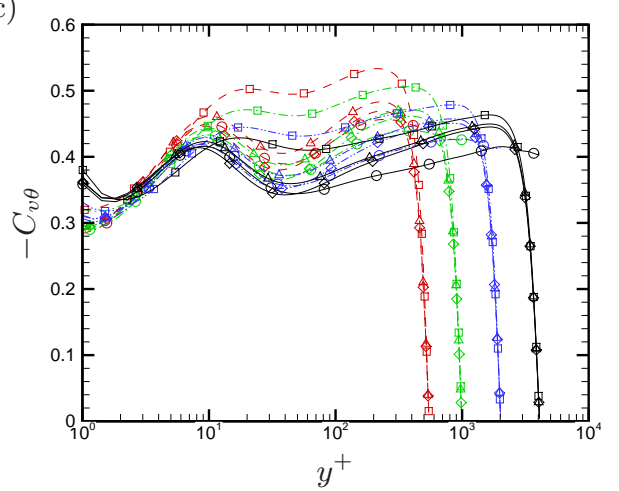

Figure 12: Correlation coefficients: $u-v(\mathrm{a}), u-\theta(\mathrm{b}), v-\theta$ (c). Refer to tables 1,2 for nomenclature of the DNS data.

fluctuations can be further analyzed in terms of the correlation coefficients, defined as

$$
C_{a b}=\frac{\overline{a^{\prime} b^{\prime}}}{\left(\overline{a^{\prime 2}} \overline{b^{\prime 2}}\right)^{1 / 2}},
$$

and shown in figure 12. As also previously noticed by Priyadarshana \& Klewicki (2004), the $u-v$ correlation coefficient stays close to 0.4 throughout the wall layer, with a slight but steady reduction as $R e_{\tau}$ increases, which may be interpreted as a reduction in the flow anisotropy. What we see here is that the $v-\theta$ correlation exhibits a similar trend with respect to the Reynolds number, and it also appears to be an increasing function of $\operatorname{Pr}$. The $u-\theta$ correlation coefficients further highlights strong correlation of streamwise velocity and scalar fluctuations, as also found in previous dedicated studies (Antonia et al. 2009). The main finding here is that, while the values in the outer layer seem to be similar for all the flow cases, the near-wall correlation is diminished in the low- $\operatorname{Pr}$ cases as the diffusion rate for momentum and scalar fields is sensibly different (Kawamura et al. 2004).

Additional details can be captured by analysing the joint pdf of the normalized velocity and scalar fluctuations. Let $\tilde{a}=a^{\prime}{\overline{a^{\prime 2}}}^{1 / 2}$ be a normalized fluctuation of variable $a$, the correlation coefficient between $a$ and $b$ is

$$
C_{a b}=\iint \tilde{a} \tilde{b} \mathrm{P}(\tilde{a}, \tilde{b}) \mathrm{d} \tilde{a} \mathrm{~d} \tilde{b} .
$$




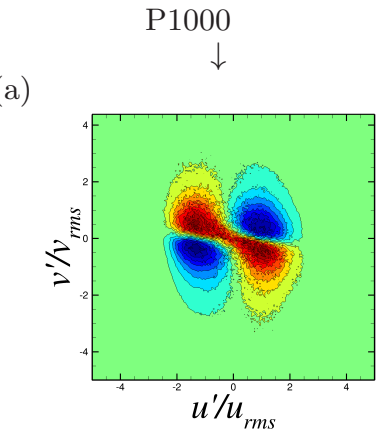

(d)

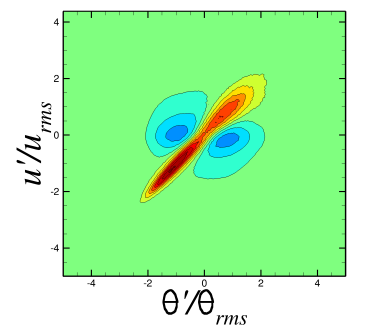

(g)

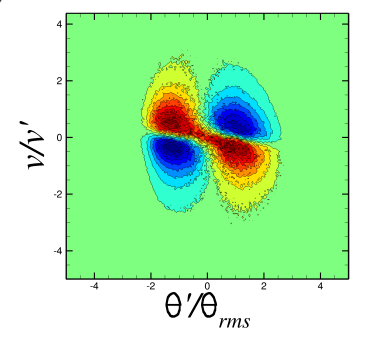

(b)

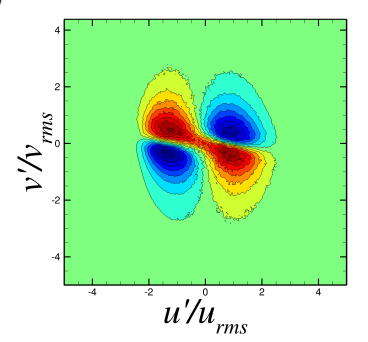

(e)

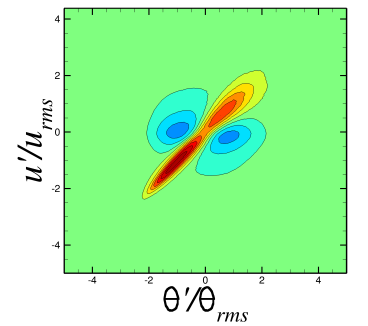

(h)

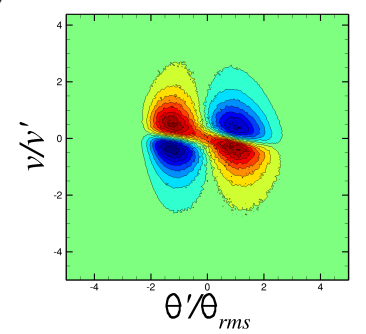

(c)

P4000

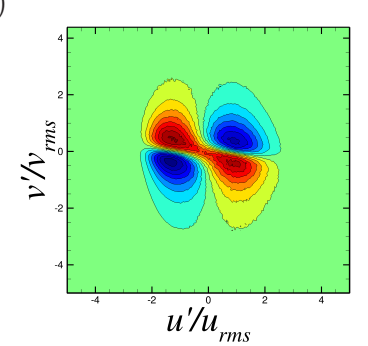

(f)

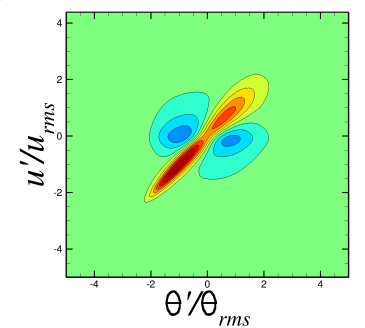

(i)

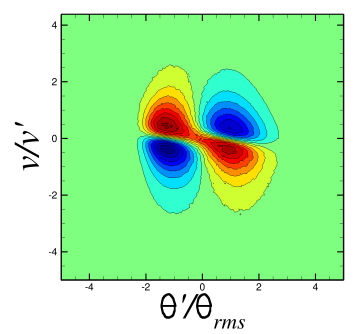

Figure 13: 'Coherent' part of joint pdfs at $y^{+}=5$, for flow case P1000 (a,d,g), P2000 (b,e,h), P4000 (c,g,i), scalar field C. Sixteen contour levels are shown, from -0.04 to 0.04 in panels $(\mathrm{a}-\mathrm{c}),(\mathrm{g}-\mathrm{i})$ and from -0.36 to 0.36 in panels $(\mathrm{d}-\mathrm{f})$. Colour scale is from blue (negative values) to red (positive values).

We then define the 'coherent' part of the joint pdf of $a$ and $b$ as

$$
\mathrm{P}^{\prime}(\tilde{a}, \tilde{b})=\mathrm{P}(\tilde{a}, \tilde{b})-\mathrm{P}(\tilde{a}) \cdot \mathrm{P}(\tilde{b}),
$$

is such a way that it is identically zero if $a$ and $b$ are statistically independent. The joint pdfs thus constructed then convey information about the local 'excess' or 'lack' of events with respect to the case of statistical independence, and of course their integrated value is zero, rather than unity. It is also straightforward to show that

$$
C_{a b}=\iint \tilde{a} \tilde{b} \mathrm{P}^{\prime}(\tilde{a}, \tilde{b}) \mathrm{d} \tilde{a} \mathrm{~d} \tilde{b}
$$

In figure 13 and 14 we show the coherent joint pdfs of velocity and scalar fluctuations at various $R e_{\tau}$, at $y^{+}=5$ and $y^{+}=100$, limited to $\operatorname{Pr}=1$ (scalar field $\mathrm{C}$ ). At $y^{+}=100$, the $u-v$ and $v-\theta$ joint pdfs indicate increased frequency of events in the second and fourth quadrant, with maxima at about one standard deviation from the mean. On the 
(a)

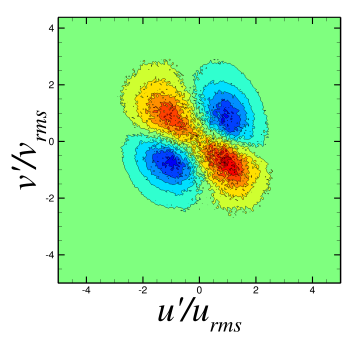

(d)

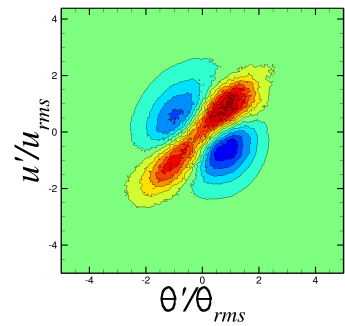

(g)

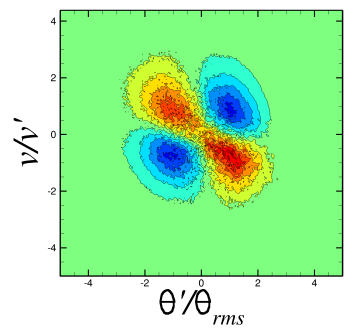

(b) P2000

$\downarrow$

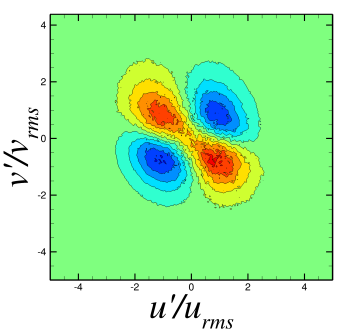

(e)

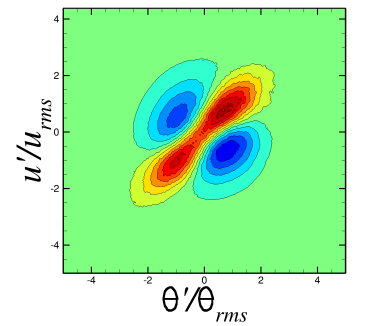

(h)

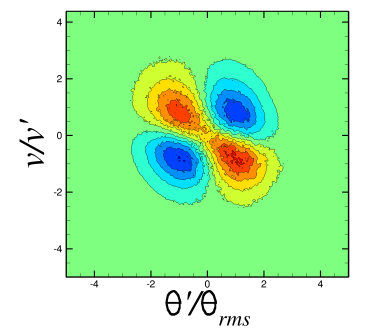

(c)

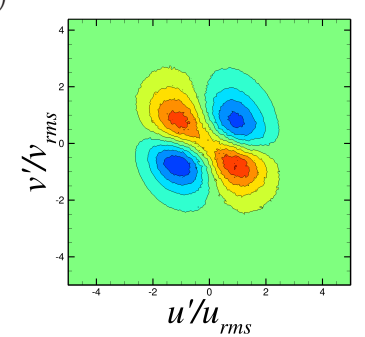

(f)

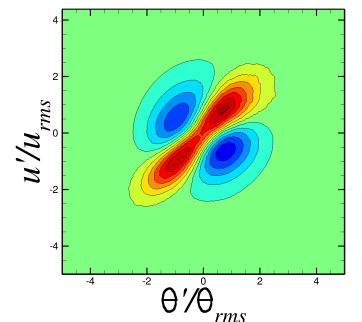

(i)

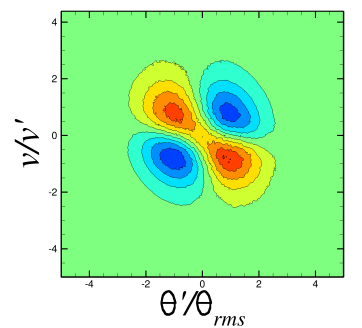

Figure 14: 'Coherent' part of joint pdfs at $y^{+}=100$, for flow case P1000 (a,d,g), P2000 (b,e,h), P4000 (c,g,i), scalar field C. Sixteen contour levels are shown, from -0.04 to 0.04 in panels (a-c),(g-i), and from -0.1 to 0.1 in panels (d-f). Colour scale is from blue (negative values) to red (positive values).

other hand, the $u-\theta$ joint pdfs show clear preference for events in the first and third quadrant, again with maximum values around one standard deviation away from the mean. A similar behavior is also recovered near the wall, where events in the $u-\theta$ plane are even more concentrated along the main diagonal, whereas events with low absolute value of $v^{\prime}$ seem to be favoured in the $u-v$ and $v-\theta$ joint pdf's. The pdf's here reported do not show visually observable hints of Reynolds number dependence.

To identify the motions responsible for the previously noticed Reynolds number variation of the correlation coefficients, in figure 15 we show the quadrant contributions to equation (3.17), in such a way that they add up to the correlation coefficient. The $u-v$ correlation (panels $(\mathrm{a}, \mathrm{b})$ ) receives positive contribution from all quadrants (unlike in the conventional quadrant analysis), with greater influence of second-quadrant motions (ejections), which are apparently responsible for the decreasing trend with $R e_{\tau}$. On the other hand, fourth-quadrant motions (sweeps) seem to be marginally affected. A very similar scenario is observed for the $v-\theta$ correlation in panels $(\mathrm{e}, \mathrm{f})$. The quadrant contri- 
(a)

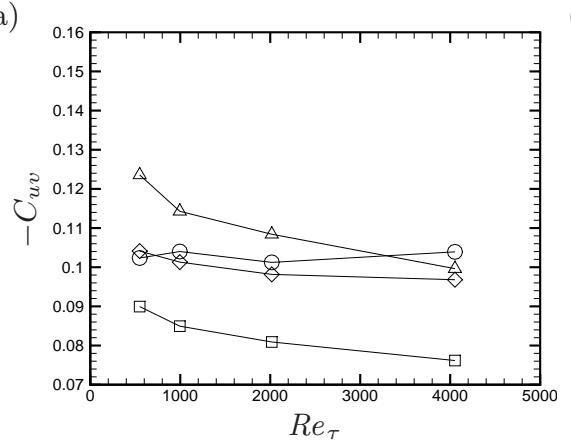

(c)

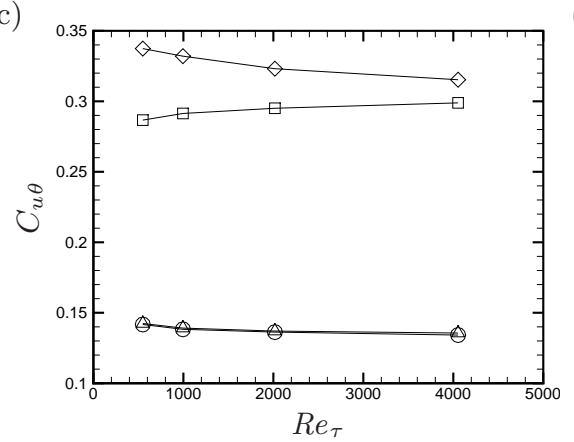

(e)

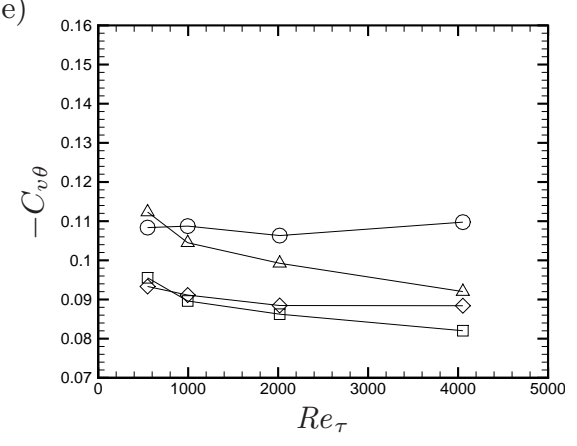

$$
y^{+}=100
$$

(b)

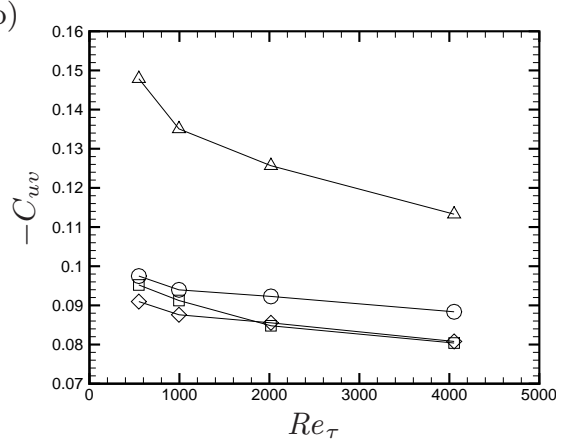

(d)

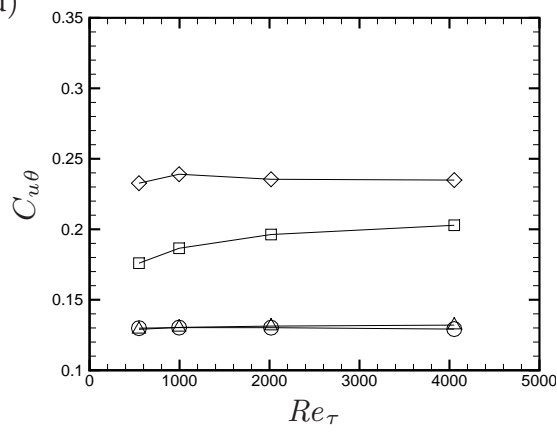

(f)

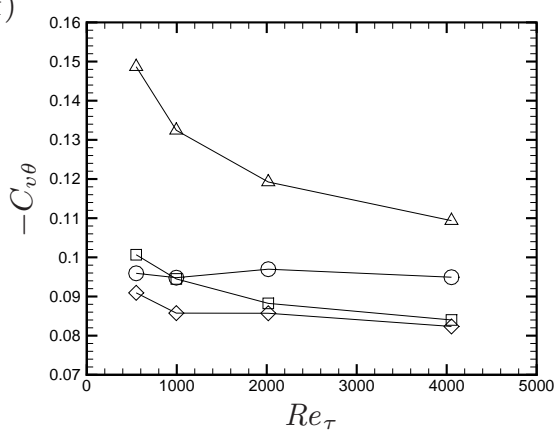

Figure 15: Quadrant contributions to correlation coefficients of $u-v(\mathrm{a}, \mathrm{b}), u-\theta(\mathrm{c}, \mathrm{d})$, $v-\theta(\mathrm{e}, \mathrm{f})$ at $y^{+}=5(\mathrm{a}, \mathrm{c}, \mathrm{e})$, and $y^{+}=100(\mathrm{~b}, \mathrm{~d}, \mathrm{f})$, for scalar field C. Quadrant I: squares; quadrant II: triangles; quadrant III: diamonds; quadrant IV: circles.

butions to the $u-\theta$ correlations, shown in panels $(\mathrm{c}, \mathrm{d})$ highlight dominant contribution from first- and especially third-quadrant events, namely events with like-signed $u$ and $\theta$ fluctuations, and insensitivity to $R e_{\tau}$.

It is noteworthy that the observed changes in the $u-v$ and $v-\theta$ correlation coefficients can be effectively compensated through normalization with the respective wall-normal velocity variance, as shown in figure 16. Compared with figure 12, it shows near universality of $-\overline{u^{\prime} v^{\prime}} / \overline{v^{\prime 2}}$ both in the inner and in the outer layer, with an overlap layer extending from $y^{+} \approx 100$ to $y / h \approx 0.5$, in which the distribution is then expected to be logarithmic (as in fact probably is), with values not far from 0.7. Concerning the scalar fields, the figures again shows universality with $R e$ in the inner and in the outer layer, 
(a)

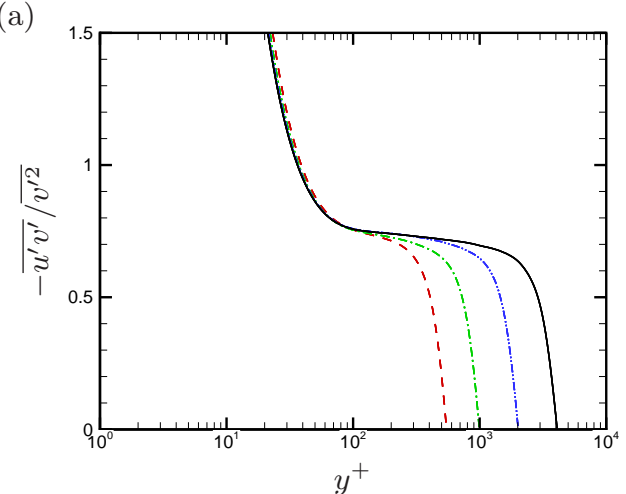

(c)

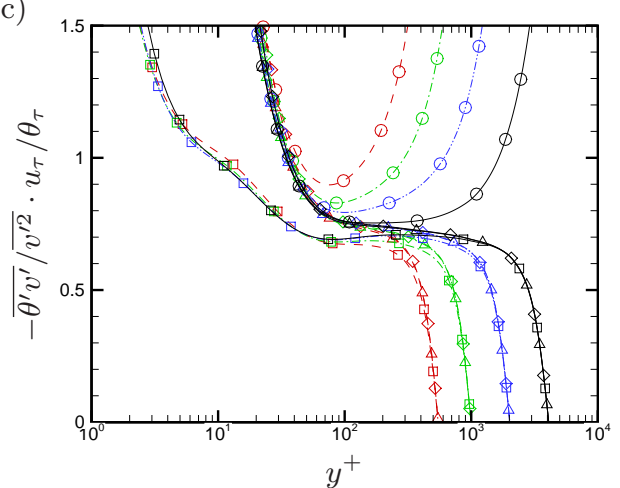

(b)

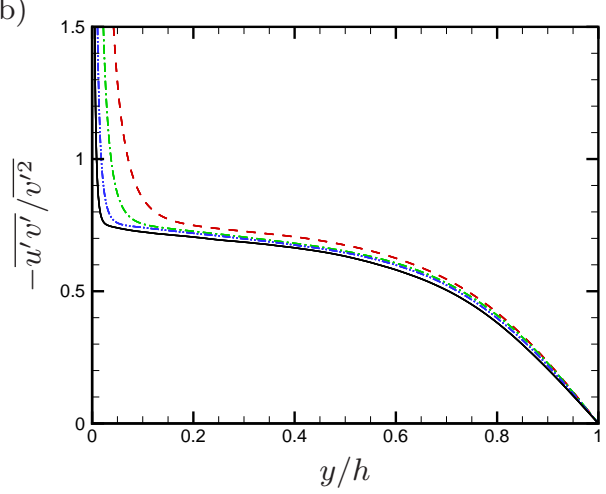

(d)

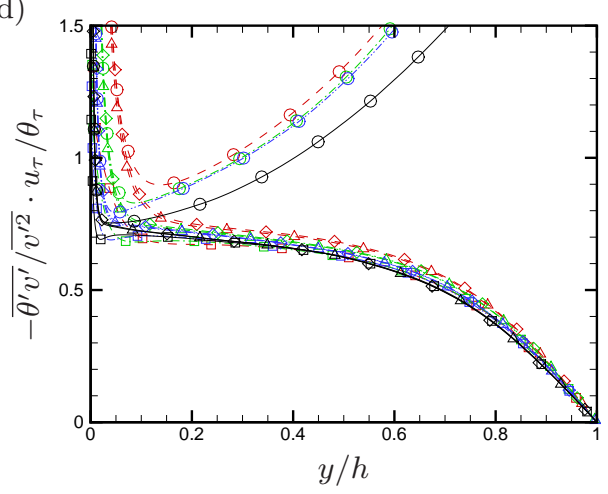

Figure 16: Ratio of turbulent shear stress (a-b) and turbulent scalar flux (c-d) to vertical velocity variance, in inner $(\mathrm{a}, \mathrm{c})$, and outer $(\mathrm{b}, \mathrm{d})$ units. Refer to tables 1,2 for nomenclature of the DNS data.

the effect of $\operatorname{Pr}$ being limited to the conductive sublayer. In the overlap layer a logarithmic distribution is clearly established for the cases with uniform forcing, with values close to those of the turbulent shear stress. The similarity between turbulent fluxes and vertical velocity fluctuations is probably not unexpected, since the production term in the evolution equation for the turbulent shear stress is proportional to the vertical velocity variance (Pope 2000). The same can be readily shown also for the vertical turbulent scalar flux. Constancy of the $u-v$ and $v-\theta$ correlation coefficients is the core of many classical turbulence models (i.e. the $k-\varepsilon$ model) is put into question by the present DNS results, hence the above information is potentially useful for the construction of improved models for turbulent scalar transport, for instance in the fashion of Durbin's $k-\varepsilon-v^{2}$ model (Durbin 1991).

\subsection{Engineering correlations}

A quantity of great importance in RANS models of scalar transport is the turbulent Prandtl number, defined as (Cebeci \& Bradshaw 1984)

$$
\operatorname{Pr}_{t}=\frac{\nu_{t}}{\alpha_{t}}=\frac{\overline{u^{\prime} v^{\prime}}}{\overline{v^{\prime} \theta^{\prime}}} \frac{\mathrm{d} \bar{\theta} / \mathrm{d} y}{\mathrm{~d} \bar{u} / \mathrm{d} y},
$$

whose distribution is shown in figure 17. Consistent with most available numerical and experimental data (Cebeci \& Bradshaw 1984; Kader 1981), for $\operatorname{Pr} \approx 1, \operatorname{Pr}_{t}$ is not far from 0.85 in a large part of the wall layer, from $y^{+} \approx 100$ to $y / h \approx 0.5$. Deviations appear 
(a)

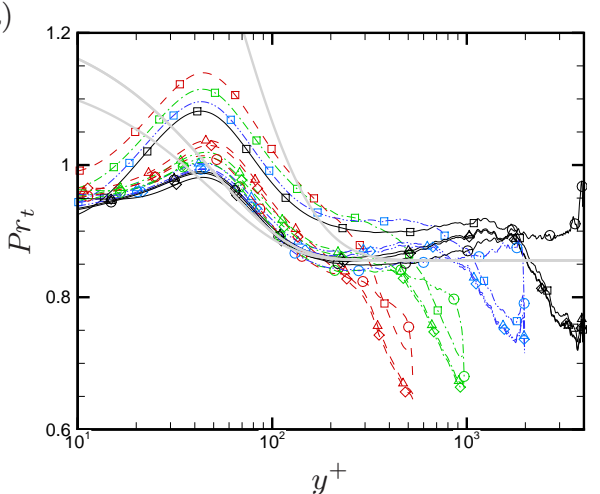

(b)

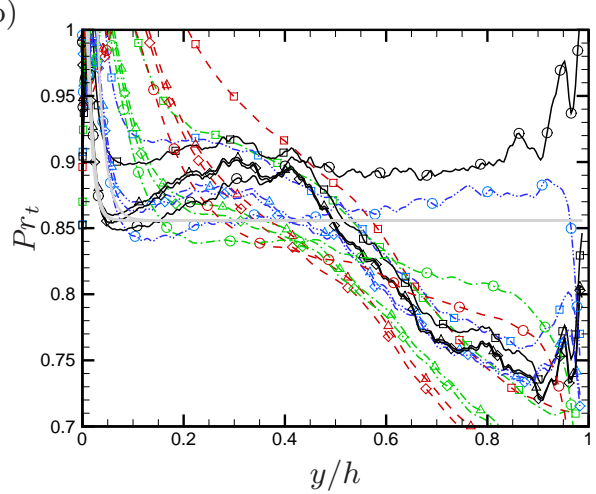

Figure 17: Turbulent Prandtl number as a function of wall distance. The thick grey lines indicate the predictions of equation (3.19) at $\operatorname{Pr}=0.2,0.71,1$. Refer to table 1 for nomenclature of the DNS data.

in case A at $\operatorname{Pr}=0.2$, for which $\operatorname{Pr}_{t}$ is closer to 0.9. Unlike previous simulations at lower Re (Kawamura et al. 2000), the turbulent Prandtl number is not found to be severely affected by the wall state, at least far from the channel centerline. Theoretical estimates for the wall-normal variation of $P r_{t}$ in the inner layer were proposed by Cebeci (1973), according to which

$$
\operatorname{Pr}_{t}=\frac{k}{k_{\theta}} \frac{1-\exp \left(-y^{+} / A\right)}{1-\exp \left(-y^{+} / B\right)}, \quad B=\frac{1}{\operatorname{Pr}^{1 / 2}} \sum_{i=1}^{5} C_{i}\left(\log _{10} \operatorname{Pr}\right)^{i-1},
$$

where $A=26, C_{1}=34.96, C_{2}=28.79, C_{3}=33.95, C_{4}=6.3, C_{5}=-1.186$. The prediction of equation (3.19), shown in grey lines in figure 17, are found not to be very accurate. It is also noteworthy that the assumption of constant $\operatorname{Pr}_{t}$ is not very accurate in the central part of the channel, where the distributions fall rather abruptly to lower values, consistent with the observations previously made about figure 2 .

Another quantity of primary engineering interest is the Nusselt number, defined as

$$
N u=\left.\frac{h}{\left(\theta_{b}-\theta_{w}\right)} \frac{\mathrm{d} \bar{\theta}}{\mathrm{d} y}\right|_{w},
$$

where $\theta_{b}=\int u \theta \mathrm{d} y / \int u \mathrm{~d} y$ is the bulk value of $\theta$, which is shown in figure 18 . As is well known (Cebeci \& Bradshaw 1984), $N u$ is found to be an increasing function of the Reynolds and the Prandtl number. It is noteworthy that scalar field D, which corresponds to the case of assigned temperature difference yields very similar (in fact, slightly smaller) Nusselt number than case B with uniform heating. In fact, it turns out that although the heat flux is larger in case D owing to more efficient redistribution of temperature in the core part of the channel by the previously noticed large eddies, the bulk temperature is also larger, the two effect very nearly compensating each other in equation (3.20). In the figure we also report predictions from the empirical fits for constant temperature walls proposed by Kays et al. (1980)

$$
N u=0.021 \operatorname{Re}_{b}^{0.8} \operatorname{Pr}^{0.5},
$$

by Sleicher \& Rouse (1975),

$$
N u=4.8+0.0156 \operatorname{Re}_{b}^{0.85} \operatorname{Pr}^{0.93},
$$




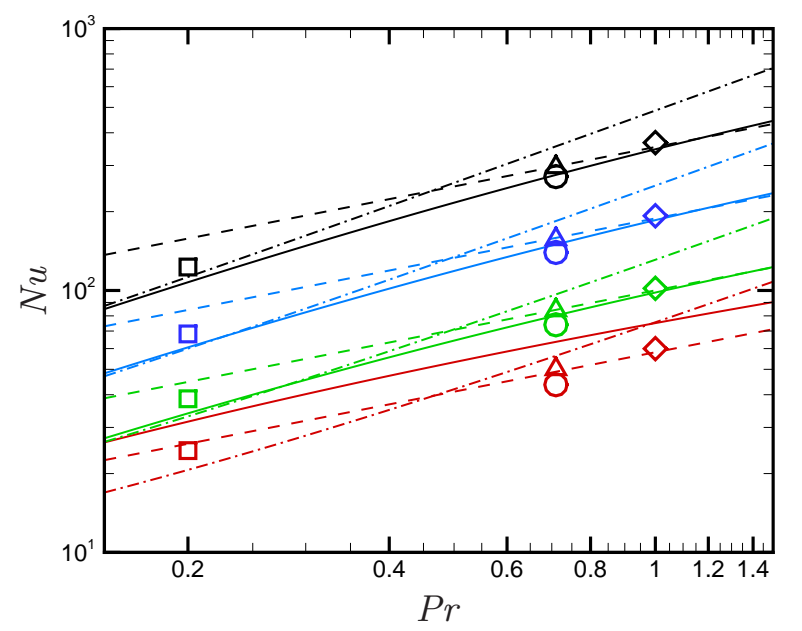

Figure 18: Nusselt number for scalar fields A-D (symbols as in table 2) at various Reynolds numbers (colors as in table 1), compared with the empirical formulas of Kays et al. (1980) (dashed lines), Sleicher \& Rouse (1975) (dot-dashed lines) Gnielinski (1976) (solid lines).

as well as the thermal analogy by Gnielinski (1976)

$$
N u=\frac{\operatorname{Pr}_{f} / 2\left(R e_{b}-1000\right)}{1+12.7\left(C_{f} / 2\right)^{1 / 2}\left(\operatorname{Pr}^{2 / 3}-1\right)},
$$

where $C_{f}$ is the skin friction coefficient. Figure 18 shows accurate prediction of Kays \& Crawford correlation at $O(1)$ Prandtl number, and superior accuracy of the formula by Sleicher Rouse at low $\mathrm{Pr}$. Gnielinski's thermal analogy performs relatively well at all $\operatorname{Pr}$ under scrutiny, and it can probably be further improved by slightly changing the additive coefficient at the numerator of equation (3.23). Consistent with the previous observations, the above correlations can be applied with the same accuracy to both the case of assigned wall temperature difference and assigned heat flux.

\section{Conclusions}

The statistics of passive scalars in turbulent channel flow have been studied by means of DNS at much higher Reynolds number than previous studies, and some previously unnoticed features have been brought to light. Upon visual analysis, the mean scalar profiles are found to qualitatively agree with semi-empirical models (Kader 1981) based on logarithmic overlap layer behavior with Prandtl-dependent shift. However, closer examination shows that, similar to what previously found for the velocity field (Bernardini et al. 2014), the scalar fields also exhibit systematic deviations from the logarithmic behavior, mainly in the form of an additional term scaling linearly with the outer wall distance, whose slope thus decreases with $R e_{\tau}$ in wall units. This 'extended' logarithmic layer occupies about $50 \%$ of each half-channel, hence it is much wider than alleged 'genuine' log layers which may arise at yet higher Reynolds number. The outer representation is particularly useful in this respect as it allows to identify with good approximation asymptotic values of the Karman constant, which is found to be $k=0.41$ for the velocity field, and $k_{\theta}=0.46$ for the scalar field, regardless of the Prandtl number and of the wall state. Outer defect representation also well highlights the presence of extended regions 
with parabolic variation of the mean scalar fields and of the velocity field in the core of forced channels, which smoothly join the quasi-logarithmic overlap profiles. It is found that the curvature of the parabolic region is higher for the velocity field, hence making up for a stronger wake region with respect to passive scalars. This observation has been traced to greater eddy diffusivity of the scalar field, which reflects in turbulent Prandtl below unity throughout the outer layer (say, $y^{+} \gtrsim 100$ ). The scalar variance fields are found to have the same qualitative behavior as the streamwise velocity variance, with the exception of the case of assigned wall difference, which also exhibits significant scalar activity toward the channel centerline. The near-wall peak in the buffer layer is inhibited at low Prandtl number, as the thickness of the conductive sublayer is comparable with the buffer layer thickness. The amplitude of the near-wall scalar variance peak at $\operatorname{Pr}=1$ appears to be higher than for the streamwise velocity field, as in the latter case redistributive effects due to pressure/strain correlation are activated. A near logarithmic layer appears away from the wall, followed by a nearly universal core region, where the scalar variances are again near parabolic. In this region the turbulence kinetic energy production term is larger than the scalar production term, hence the streamwise velocity fluctuations are stronger than the scalar fluctuations, which is the likely reason why a 'bump' is observed in the velocity variances rather than a genuine logarithmic layer, at least at these Reynolds numbers. Throughout the wall layer the scalar dissipation is found to be significantly larger than the streamwise velocity dissipation, which implies that the smallest scalar-bearing eddies are more energetic, as confirmed by inspection of their energy spectra. This is also reflected in the instantaneous flow visualizations, which show sharper fronts of the scalar-bearing eddies as compared to those associated with the streamwise velocity field, although visual correspondence of the two fields is clear, and their correlation coefficient remains higher than 0.6 across large part of the wall layer. Reynolds number effects manifest themselves in the decrease of the $u-v$ and $u-\theta$ correlation coefficients in the outer layer, which is an indication that inactive motions become comparatively more important. An in-depth analysis has shown that the decrease is mainly related to decreased contribution of the second-quadrant motions (ejections), and it can be compensated by normalization with the vertical velocity variance. From an engineering standpoint, our high-Re data show that the turbulent Prandtl number remains in the range between 0.85 and 0.9 in the lower $50 \%$ of the wall layer, and it decreases almost linearly toward the centerline, where $P r_{t} \approx 0.7$. The Nusselt number follows the classical increasing behavior with $R e_{\tau}$ and $P r$, and it is in good agreement with established thermal analogies. Open issues include the detailed assessment of the scalar forcing strategy, which is found to have some effect on the statistical properties in the core part of the flow. Small but systematic differences with respect to old correlations for the mean scalar profiles (Kader 1981) also deserve further investigation.

Flow statistics are available at the web page http://newton.dma. uniroma1.it/scalars/, with supporting documentation.

We acknowledge that the results reported in this paper have been achieved using the PRACE Research Infrastructure resource FERMI based at CINECA, Casalecchio di Reno, Italy.

\section{REFERENCES}

Abe, H. \& Antonia, R.A. 2009 Near-wall similarity between velocity and scalar fluctuations in a turbulent channel flow. Phys. Fluids 21, 025109.

Abe, H., Kawamura, H. \& Choi, H. $2004 a$ Very large-scale structures and their effects on the 
wall shear-stress fluctuations in a turbulent channel flow up to re $\tau=640$. J. Fluids Eng. 126, 835-843.

Abe, H., Kawamura, H. \& Matsuo, Y. $2004 b$ Surface heat-flux fluctuations in a turbulent channel flow up to $\operatorname{Re}_{\tau}=1020$ with $\mathrm{Pr}=0.025$ and 0.71. Int. J. Heat Fluid Flow 25, 404-419.

AfZAL, N. \& YAJniK, K. 1973 Analysis of turbulent pipe and channel flows at moderately large Reynolds number. J. Fluid Mech. 61, 23-31.

Del Álamo, J.C. \& JimÉnez, J. 2003 Spectra of the very large anisotropic scales in turbulent channels. Phys. Fluids 15, L41-L44.

del Álamo, J. C., Jiménez, J., Zandonade, P. \& Moser, R. D. 2004 Scaling of the energy spectra of turbulent channels. J. Fluid Mech. 500, 135-144.

Antonia, R.A., Abe, H. \& Kawamura, H. 2009 Analogy between velocity and scalar fields in a turbulent channel flow. J. Fluid Mech. 628, 241-268.

BAtchelor, G.K. 1959 Small-scale variation of convected quantities like temperature in turbulent fluid Part 1. general discussion and the case of small conductivity. J. Fluid Mech. 5, 113-133.

Bernardini, M, Pirozzoli, S \& Orlandi, P 2014 Velocity statistics in turbulent channel flow up to $\operatorname{Re}_{\tau}=4000$. J. Fluid Mech. 742, 171-191.

Bernardini, M, Pirozzoli, S, Quadrio, M \& Orlandi, P 2013 Turbulent channel flow simulations in convecting reference frames. J. Comput. Phys. 232, 1-6.

Cebeci, T. 1973 A model for eddy conductivity and turbulent Prandtl number. J. Heat Transfer 95, 227-234.

Cebeci, T. \& Bradshaw, P. 1984 Physical and computational aspects of convective heat transfer. Springer-Verlag, New York, NY.

DeGraAfF, D.B. \& Eaton, J.K. 2000 Reynolds-number scaling of the flat-plate turbulent boundary layer. J. Fluid Mech. 422, 319-346.

Durbin, P.A. 1991 Near-wall turbulence closure modeling without damping functions. Theoret. Comput. Fluid Dyn. 3, 1-13.

Gnielinski, V. 1976 New equations for heat and mass transfer in turbulent pipe and channel flow. Int. Chem. Eng. 16, 359-367.

Gowen, R.A. \& Smith, J.W. 1967 The effect of the Prandtl number on temperature profiles for heat transfer in turbulent pipe flow. Chem. Eng. Science 22, 1701-1711.

Hutchins, N. \& MARusic, I. 2007 Evidence of very long meandering features in the logarithmic region of turbulent boundary layers. J. Fluid Mech. 579, 1-28.

Hutchins, N., Nickels, T.B., Marusic, I. \& Chong, M.S. 2009 Hot-wire spatial resolution issues in wall-bounded turbulence. J. Fluid Mech. 635, 103-136.

Jiménez, J. \& Pinelli, A. 1999 The autonomous cycle of near-wall turbulence. J. Fluid Mech. 389, 335-359.

KADER, B.A. 1981 Temperature and concentration profiles in fully turbulent boundary layers. Int. J. Heat Mass Transfer 24, 1541-1544.

Kawamura, H., Abe, H. \& Matsuo, Y. 1999 DNS of turbulent heat transfer in channel flow with respect to Reynolds and Prandtl number effects. Int. J. Heat Fluid Flow 20, 196-207.

Kawamura, H., Abe, H. \& Matsuo, Y. 2004 Very large-scale structures observed in dns of turbulent channel flow with passive scalar transport. In Proc. 15th Australasian Fluid Mechanics Conference, pp. 15-32.

Kawamura, H., ABE, H. \& Shingai, K. 2000 DNS of turbulence and heat transport in a channel flow with different Reynolds and Prandtl numbers and boundary conditions. In Proc. 3rd Int. Symp. on Turbulence, Heat and Mass Transfer (ed. Y. Nagano), pp. 15-32.

Kawamura, H., Ohsaka, K., Aвe, H. \& Yamamoto, K. 1998 DNS of turbulent heat transfer in channel flow with low to medium-high Prandtl number. Int. J. Heat Fluid Flow 19, 482-491.

Kays, W.M., Crawford, M.E. \& Weigand, B. 1980 Convective heat and mass transfer. McGraw-Hill.

Kim, J. \& Moin, P. 1985 Application of a fractional-step method to incompressible NavierStokes equations. J. Comput. Phys. 59, 308-323.

Kim, J. \& Moin, P. 1989 Transport of passive scalars in a turbulent channel flow. In Turbulent Shear Flows 6, pp. 85-96. Springer. 
Kim, J., Moin, P. \& Moser, R.D. 1987 Turbulence statistics in fully developed channel flow at low Reynolds number. J. Fluid Mech. 177, 133-166.

Kleiser, L. \& ZANG, T.A. 1991 Numerical simulation of transition in wall-bounded shear flows. Annu. Rev. Fluid Mech. 23, 495-537.

Lee, M. \& Moser, R.D. 2015 Direct simulation of turbulent channel flow layer up to $\operatorname{Re}_{\tau}=$ 5200. J. Fluid Mech. 774, 395-415.

Lyons, S.L., Hanratty, T.J. \& McLaughlin, J.B. 1991 Direct numerical simulation of passive heat transfer in a turbulent channel flow. Int. J. Heat Mass Transfer 34, 11491161.

Monin, A.S. \& Yaglom, A.M. 1971 Statistical fluid mechanics: mechanics of turbulence, , vol. 1. MIT Press, Cambridge MA.

Moser, R.D., Kim, J. \& Mansour, N.N. 1999 Direct numerical simulation of turbulent channel flow up to $\operatorname{Re}_{\tau}=590$. Phys. Fluids 11, 943-945.

NAGANO, Y. \& TAGAWA, M. 1988 Statistical characteristics of wall turbulence with a passive scalar. J. Fluid Mech. 196, 157-185.

OrLANDI, P. 2000 Fluid flow phenomena: a numerical toolkit. Kluwer.

Orlandi, P., Bernardini, M. \& Pirozzoli, S. 2015 Poiseuille and Couette flows in the transitional and fully turbulent regime. J. Fluid Mech. pp. 424-441.

Perry, A.E. \& Marusic, I. 1995 A wall-wake model for the turbulence structure of boundary layers. part 1. extension of the attached eddy hypothesis. J. Fluid Mech. 298, 361.

Pirozzoli, S. 2014 Revisiting the mixing-length hypothesis in the outer part of turbulent wall layers: mean flow and wall friction. J. Fluid Mech. 745, 378-397.

Pirozzoli, S., Bernardini, M. \& Orlandi, P. 2014 Turbulence statistics in Couette flow at high Reynolds number. J. Fluid Mech. 758, 327-343.

Pope, S.B. 2000 Turbulent flows. Cambridge University Press.

Priyadarshana, P.J.A. \& Klewicki, J.C. 2004 Study of the motions contributing to the Reynolds stress in high and low reynolds number turbulent boundary layers. Phys. Fluids 16, 4586-4600.

Schwertfirm, F. \& Manhart, M. 2007 DNS of passive scalar transport in turbulent channel flow at high Schmidt numbers. Int. J. Heat Fluid Flow 28, 1204-1214.

Sleicher, C.A. \& Rouse, M.W. 1975 A convenient correlation for heat transfer to constant and variable property fluids in turbulent pipe flow. Int. J. Heat Mass Transfer 18, 677-683.

Subramanian, C.S. \& Antonia, R.A. 1981 Effect of Reynolds number on a slightly heated turbulent boundary layer. Int. J. Heat Mass Transfer 24, 1833-1846.

Tennekes, H. \& Lumley, J. L. 1972 A first course in turbulence. MIT Press.

Townsend, A.A. 1976 The Structure of Turbulent Shear Flow. 2nd edn. Cambridge University Press.

Wikström, P.M. \& Johansson, A.V. 1998 DNS and scalar-flux transport modelling in a turbulent channel flow. In Proc. the 2nd EF Conference in Turbulent Heat Transfer, pp. $6.46-6.51$. 